\title{
Duality in Mean-Variance Frontiers with Conditioning Information*
}

\author{
Francisco Peñaranda \\ UPF, Ramon Trias Fargas 25-27, E-08005 Barcelona, Spain. \\ $<$ francisco.penaranda@upf.edu> \\ Enrique Sentana \\ CEMFI, Casado del Alisal 5, E-28014 Madrid, Spain. \\ <sentana@cemfi.es>
}

October 2007

\begin{abstract}
Portfolio and stochastic discount factor (SDF) frontiers are usually regarded as dual objects, and researchers sometimes use one to answer questions about the other. However, the introduction of conditioning information and active portfolio strategies alters this relationship. For instance, the unconditional portfolio frontier in Hansen and Richard (1987) is not dual to the unconditional SDF frontier in Gallant, Hansen and Tauchen (1990). We characterise the dual objects to those frontiers, and relate them to the frontiers generated with managed portfolios, which are commonly used in empirical work. We also study the implications of a safe asset and other special cases.
\end{abstract}

Keywords: Asset Pricing, Dynamic Portfolio Strategies, Representing portfolios, Stochastic Discount Factors.

JEL: G11, G12

\footnotetext{
${ }^{*}$ We would like to thank Torben Andersen, Tony Berrada, Wayne Ferson, Lars Hansen, Jens Jackwerth and Ravi Jagannathan for useful comments and discussions, as well as seminar audiences at Kellogg, UPF, EFA (Ljubljana, 2007) and ESEM (Budapest, 2007). Of course, the usual caveat applies. Financial support from the Spanish Ministry of Education and Science through grants SEJ2005-02829 (Peñaranda) and SEJ2005-08880 (Sentana) is gratefully acknowledged.
} 


\section{Introduction}

Mean-variance analysis continues to be widely used in economics and finance, with applications that cover such key issues as portfolio choice, asset pricing tests and performance evaluation. In this sense, finance students nowadays learn that there is not just one, but two types of mean-variance frontiers: one for portfolios due to Markowitz (1952), and another one for stochastic discount factors (SDFs), due to Hansen and Jagannathan (1991). They learn that the first frontier characterises the risk-return trade-offs that an investor faces, while the second one describes the mean-variance constraints that financial markets data imposes on asset pricing models. $^{1}$

Students also learn that asset returns are predictable, if not in mean at least in variance, and that investors can exploit this fact to their advantage by using conditional distributions as opposed to unconditional ones in designing their portfolio strategies. ${ }^{2}$ For instance, an investor can not only choose a passive "buy and hold" portfolio strategy whose weights are fixed over time, but also define a dynamic trading strategy as a function of the volatility level of the stock market. As a result, more advanced students learn that there are different versions of the return and SDF mean variance frontiers, depending on the information used in their construction. Frontiers for such active strategies were introduced by Hansen and Richard (1987) in the case of portfolios, and Gallant, Hansen and Tauchen (1990) for SDFs, and have been recently revisited by Ferson and Siegel (2001, 2003, 2006), Bekaert and Liu (2004), and Abhyankar, Basu and Stremme (2007).

This paper systematises and extends our knowledge on the precise relationship between mean-variance frontiers across both these dimensions, namely type (i.e. portfolio vs SDF) and information. This is an important issue because portfolio and stochastic discount factor frontiers are usually regarded as dual objects (in the sense that every element in one frontier is believed to have a counterpart in the other one), to the extent that sometimes researchers use one type of frontier to answer questions that arise more naturally in the other type. For instance, De Santis (1995) and Bekaert and Urias (1996) assess the gains for a mean-variance investor from internationally diversifying her portfolio by testing if the restrictions that domestic market data imposes on asset pricing models are strengthened by the inclusion of data on foreign assets. ${ }^{3}$

\footnotetext{
${ }^{1}$ In line with most of the literature, in this paper we do not consider SDF frontiers that impose positivity of the SDF. See Hansen and Jagannathan (1991) for details.

${ }^{2}$ See Cochrane (2001) for a summary of the empirical evidence on mean predictability, and Sentana (2005) for a recent example of the link between regression forecasts and optimal portfolios.
} 
Similarly, Cochrane (2001, sec. 21.1) uses unconditional Sharpe ratios of traded assets to infer the volatility of the SDF required to explain the equity premium puzzle with a consumptionbased asset pricing model.

Nevertheless, it is important to remember that the widely cited duality result in Hansen and Jagannathan (1991) applies to their specific set-up: unconditional moments of passive strategies based on a given vector of asset payoffs. Moreover, it is also important to bear in mind that the portfolio frontier that they consider may only have unit cost on average, as the prices of the asset payoffs under consideration may depend on the information available at the time of trading. In that case, the Hansen-Jagannathan portfolio frontier will differ from the usual Markowitz frontier for returns, which is the relevant object from the perspective on an uninformed investor.

In this paper, we first show that the conditional portfolio frontier in Hansen and Richard (1987) is dual to the conditional SDF frontier in Gallant, Hansen and Tauchen (1990), both of which refer to conditional moments of active strategies. In contrast, we show that duality usually fails when we work with unconditional moments of active portfolios. Specifically, we show that the unconditional frontiers in those papers are not dual objects, so that the questions on investors' risk-return trade-offs and constraints on asset pricing models that they respectively answer are not necessarily equivalent either. In this context, we explicitly characterise the random variables for which the appropriate dual objects are themselves frontiers, as opposed to mere volatility bounds. An important implication of our results is that empirical researchers should be careful, and focus on the type of frontier that is really relevant for the particular question that they want to address.

In order to avoid the misspecification of a conditional model for asset payoffs, the most popular empirical strategy to construct unconditional mean-variance frontiers approximates the effect of conditioning information by constructing passive frontiers with managed portfolios, i.e., portfolios whose scale is a function of some variables in the econometrician's information set. For that reason, we will also relate the mean-variance frontiers that such a procedure generates to the frontiers mentioned in the previous paragraph. Our analysis implies that an empirical researcher who is interested in SDF frontiers should use unrestricted managed portfolios, while a researcher who is interested in portfolio frontiers should use managed portfolios of constant cost instead.

\footnotetext{
${ }^{3}$ See De Roon and Nijman (2001) for a survey on spanning tests, including a review of their implementation under conditioning information.
} 
Table 1 summarises our analysis. Columns are arranged by a decreasing use of conditioning information, while for each column the last two rows couple the appropriate dual frontiers.

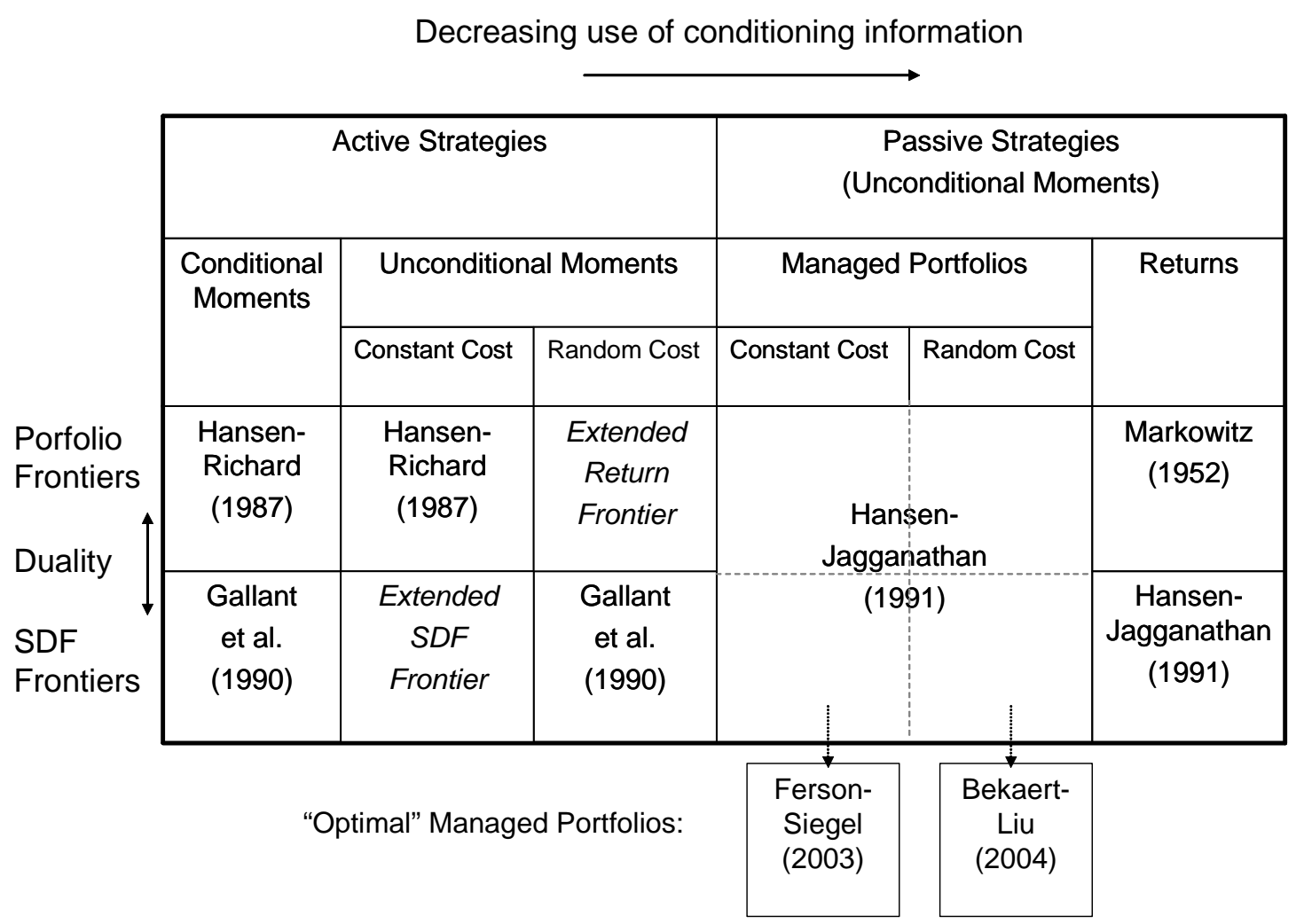

Table 1: Mean-variance frontiers across type and information.

Finally, we also study some special cases of simplified mean-variance frontiers, the most important of which arises in the presence of an asset that is either conditionally or unconditionally riskless. In that case, we show that the geometric interpretation of duality in terms of Sharpe ratios in Hansen and Jagannathan (1991) applies once again to their specific set-up (i.e. unconditional moments of passive strategies), so that one must be careful in extending their result to other frameworks. We also show that some other results that are sometimes taken for granted may fail too. For instance, while it is true that portfolio mean-variance frontiers with and without a safe asset share the so-called tangency portfolio when we work with either unconditional moments of passive strategies, or conditional moments of active strategies, no tangency portfolio exists any longer when we work with unconditional moments of active portfolios. This result is relevant for the correct interpretation of the Sharpe ratios used in some recent papers on mean-variance frontiers with conditioning information. 
The rest of the paper is organised as the columns of Table 1. We introduce the general theoretical set-up in section 2. Then, we study conditional and unconditional mean-variance frontiers in sections 3 and 4 , respectively. In section 5, we introduce what we call extended mean-variance frontiers, which are the correct dual objects to the unconditional frontiers of active portfolios. Next, we discuss passive frontiers with and without managed portfolios in section 6. Finally, we extend our analysis in the presence of a riskless asset and other special cases in sections 7 and 8, respectively, and present our conclusions in section 9. Proofs are gathered in the appendix.

\section{Theoretical Background}

\subsection{Information Structure and Active Portfolio Strategies}

Consider an economy with a finite number $N$ of risky assets whose random payoffs $\mathbf{x}=$ $\left(x_{1}, \ldots, x_{N}\right)^{\prime}$ are defined on an underlying probability space. These payoffs may correspond to stocks, bonds, derivatives, mutual funds, etc. To incorporate conditional information, we closely follow Hansen and Richard (1987), where further details can be found. Specifically, we assume that there are three important dates in this economy: $\mathcal{O}, \mathbf{l}$ and 2 . We identify $\mathcal{O}$ as the decision date, 1 as the trading date and 2 as the payoff date. Investors design ex ante portfolio strategies at 0 that may depend on the information that they will observe at 1 , when trading takes place. Finally, they receive payoffs at 2 .

Let $G_{1}$ denote the investors' information at date 1 . We will typically think of $G_{1}$ as containing one or more signals observed at 1 that are informative about future asset payoffs (see section 2.2 for an example). We denote the set of all random variables that are measurable with respect to $G_{1}$ by $I_{1}$, while $G_{2}$ and $I_{2}$ have a similar interpretation with reference to date 2.

In this context, we denote the first two conditional moments of the primitive payoffs and their conditional costs by

$$
E\left(\mathbf{x} \mid G_{1}\right)=\nu_{1}, \quad E\left(\mathbf{x x}^{\prime} \mid G_{1}\right)=\boldsymbol{\Gamma}_{1}, \quad C\left(\mathbf{x} \mid G_{1}\right)=\mathbf{c}_{1},
$$

respectively, all of which belong to $I_{1}$. To avoid a trivial uninformative set up, we assume that not all these random variables are degenerate. We also assume that the diagonal elements of $\boldsymbol{\Gamma}_{1}$ are uniformly bounded with probability one (a.s.), so that a fortiori all the elements of $\mathbf{x}$ belong to $L^{2}$, which is the collection of all random variables defined on the underlying probability space 
with bounded (unconditional) second moments. ${ }^{4}$ Hence, we can obtain the covariance matrix of $\mathrm{x}$ as $\boldsymbol{\Sigma}_{1}=\boldsymbol{\Gamma}_{1}-\boldsymbol{\nu}_{1} \boldsymbol{\nu}_{1}^{\prime}$, whose smallest eigenvalue we initially assume is uniformly bounded away from 0 a.s., which implies that none of the primitive assets is either conditionally riskless or redundant, and moreover, that it is not possible to generate a conditionally riskless portfolio from $\mathbf{x}$ other than the trivial one.

Although we deliberately allow asset prices $\mathbf{c}_{\mathbf{1}}$ to depend on the values of the signals, there are two important examples of payoffs whose costs are non-random: gross returns, which are payoffs with unit prices, and arbitrage portfolios, or zero-cost payoffs. Obviously, any payoff with a nonzero cost can be normalised to be a gross return, but the scaling factor may be a function of $G_{1}$. For simplicity, though, we exclude the possibility that all primitive assets are arbitrage portfolios by assuming that the vector $\mathbf{c}_{1}$ has at least one entry different from 0 a.s. We also assume that not all expected payoffs are conditionally proportional to their prices with a common factor of proportionality. In this way, we implicitly rule out those situations in which all conditionally expected returns are the same. ${ }^{5}$

We denote the unconditional counterparts to (1) as

$$
E(\mathbf{x})=E\left(\boldsymbol{\nu}_{1}\right)=\boldsymbol{\nu}, \quad E\left(\mathbf{x x}^{\prime}\right)=E\left(\boldsymbol{\Gamma}_{1}\right)=\boldsymbol{\Gamma}, \quad C(\mathbf{x})=E\left(\mathbf{c}_{1}\right)=\mathbf{c}
$$

which are now real numbers instead of random variables. Following Hansen and Richard (1987), we will often use the term pseudo-prices to refer to average costs.

As we said before, investors can condition their portfolios weights on the information they know they will have at the time of trading, which is given by $G_{1}$. For instance, investors may prefer different portfolios depending on whether yield spreads at date 1 are high or low. Consequently, they can construct active portfolio strategies with payoffs $p=\mathbf{x}^{\prime} \mathbf{w}_{\mathbf{l}}$, where the portfolio weights $\mathbf{w}_{1} \in I_{1}$. In what follows, we will refer to the payoff space defined by

$$
P_{a}=\langle\mathbf{x}\rangle_{1}=\left\{p \in I_{2}: p=\mathbf{x}^{\prime} \mathbf{w}_{\mathbf{1}}, \quad \mathbf{w}_{\mathbf{1}} \in I_{1}\right\}
$$

as the active payoff space, where $\langle\mathbf{x}\rangle_{1}$ denotes the conditional span of $\mathbf{x}$, which includes its unconditional span $\langle\mathbf{x}\rangle=\left\{p \in I_{2}: p=\mathbf{x}^{\prime} \mathbf{w}, \mathbf{w} \in \mathbb{R}^{N}\right\}$.

Trivially, the conditional moments and costs of the elements of $P_{a}$ will be

$$
E\left(p \mid G_{1}\right)=\mathbf{w}_{1}^{\prime} \nu_{1}, \quad E\left(p^{2} \mid G_{1}\right)=\mathbf{w}_{1}^{\prime} \boldsymbol{\Gamma}_{1} \mathbf{w}_{1}, \quad C\left(p \mid G_{1}\right)=\mathbf{w}_{1}^{\prime} \mathbf{c}_{1},
$$

\footnotetext{
${ }^{4}$ Such a restriction on conditional moments is stronger than required for our conditional analysis in section 3 , but it allows us to work with unconditional moments in later sections.

${ }^{5}$ The special cases of a riskless asset, arbitrage portfolios, and equal expected returns can also be analysed in our set up, but for pedagogical reasons we postpone them to sections 7 and 8 of the paper.
} 
all of which belong to $I_{1}$. Similarly, their unconditional moments and average cost will be the real numbers

$$
E(p)=E\left(\mathbf{w}_{\mathbf{1}}^{\prime} \boldsymbol{\nu}_{\mathbf{1}}\right), \quad E\left(p^{2}\right)=E\left(\mathbf{w}_{\mathbf{1}}^{\prime} \boldsymbol{\Gamma}_{\mathbf{1}} \mathbf{w}_{\mathbf{1}}\right), \quad C(p)=E\left(\mathbf{w}_{\mathbf{1}}^{\prime} \mathbf{c}_{\mathbf{1}}\right) .
$$

\section{$2.2 \quad$ A Multinomial Example}

From the perspective of date $\boldsymbol{o}$, the payoff space at date 2 will generally be infinite dimensional, even though investors only have access to a finite set of primitive asset payoffs, because they can use any piece of information known at date 1 in designing their investment strategies. For pedagogical reasons though, in this section we will illustrate the previous concepts in a situation in which the dimension of $P_{a}$ is finite. We will do so by particularising our analysis to the special case of an information set effectively characterised by a multinomial random variable.

Such a set up will arise, for instance, if the investor can observe two signals at date 1 , each of which can only take two values. As in most of the figures that illustrate this paper, if we start from a vector $\mathbf{x}$ that represents gross returns (whose nonrandom $\mathbf{c}_{\mathbf{1}}$ is equal to a vector of ones), the first signal could reveal one of two possible expected return vectors, while the second signal could indicate one of two possible covariance matrices. In such a context, we can always understand the investor's information set $G_{1}$ as containing a single multinomial random variable $z$ that can take the following four values

$$
\begin{array}{lll}
\text { Signals } 1 \backslash 2 & \text { Low } & \text { High } \\
\text { Low } & z=1 & z=2 \\
\text { High } & z=3 & z=4
\end{array}
$$

whose probabilities as of time $\mathcal{O}$ we shall denote by $\pi^{k}, k=1,2,3,4$, with $\sum_{k=1}^{4} \pi^{k}=1$.

Let us define the dummy variables

$$
\xi^{k}=I(z=k), \quad k=1,2,3,4,
$$

where $I(\cdot)$ is the usual indicator function. The key feature of a multinomial set-up is that

$$
P_{a}=\left\{p \in I_{2}: p=\mathbf{x}^{\prime}\left(\sum_{k=1}^{4} \xi^{k} \mathbf{w}_{1}^{k}\right)=\sum_{k=1}^{4}\left(\xi^{k} \mathbf{x}\right)^{\prime} \mathbf{w}_{1}^{k}, \quad \mathbf{w}_{1}^{k} \in \mathbb{R}^{N}\right\} .
$$

where $\mathbf{w}_{1}^{k}$ denotes the four possible values that $\mathbf{w}_{\mathbf{1}}$ may take at date $\mathbf{1}$. As a result, the payoff space $P_{a}$ is indeed finite dimensional from the point of view of date $\boldsymbol{o}$ since it could be generated by passive strategies on an augmented but finite dimensional set of managed portfolios whose 
payoffs take the form $\xi^{k} \mathbf{x}(k=1, \ldots, 4)$. In this sense, we say that a portfolio strategy is passive if the four vectors $\mathbf{w}_{1}^{k}$ are equal; otherwise, we say that it is active.

It is also very easy to obtain expressions for the mean, second moment and cost of a portfolio in a multinomial set up. For instance, while the conditional cost of a portfolio with weights $\mathbf{w}_{\mathbf{I}}$ will be given by

$$
C(p \mid z=k)=\mathbf{w}_{1}^{k \prime} \mathbf{c}_{1}^{k}, \quad k=1,2,3,4,
$$

where $\mathbf{c}_{1}^{k} \in \mathbb{R}^{N}$ denotes the four possible values that $\mathbf{c}_{1}$ may take at date $\mathbf{l}$, its unconditional cost is

$$
C(p)=\sum_{k=1}^{4} \pi^{k} \mathbf{w}_{\mathbf{1}}^{k \prime} \mathbf{c}_{\mathbf{1}}^{k} .
$$

Nevertheless, it is important to bear in mind that an investor is always concerned with the four possible values of $C(p \mid z=k)$, i.e. the cost of her portfolio at every possible realisation of $z$, and not simply its average $C(p)$. In particular, if she is endowed with some positive wealth at date 1 , which we can normalize to 1 without loss of generality, then she will only be interested in portfolio strategies that cost 1 at date $\perp$ for every possible value of $z$. From the point of view of such an investor, a strategy whose cost is only 1 on average will be either infeasible or suboptimal, as its real cost will be higher than her wealth for some values of $z$, and lower for others.

\subsection{Representing Portfolios and Stochastic Discount Factors}

Hansen and Richard (1987) introduce a conditional analogue to a standard Hilbert space based on the mean square inner product, $E\left(x y \mid G_{1}\right)$, and the associated mean square norm $\sqrt{E\left(x^{2} \mid G_{1}\right)}$, where $x, y \in L_{2}^{2}$, and $L_{2}^{2}$ is the conditional analogue to $L^{2}$. Such a topology allows them to define the conditional least squares projection of any $y \in L_{2}^{2}$ onto $P_{a}$ as

$$
E\left(y \mathbf{x}^{\prime} \mid G_{1}\right) E^{-1}\left(\mathbf{x x}^{\prime} \mid G_{1}\right) \mathbf{x}
$$

which is the element of $P_{a}$ that is closest to $y$ in the conditional mean square norm.

In this context, we can formally understand $C\left(\cdot \mid G_{1}\right)$ and $E\left(\cdot \mid G_{1}\right)$ as conditionally continuous linear functionals that map the elements of $P_{a}$ onto $I_{1}$. The expected value functional is always conditionally continuous on $L_{2}^{2}$ by a conditional version the Markov inequality. Similarly, our full rank assumption on $\boldsymbol{\Sigma}_{1}$ implies that $\boldsymbol{\Gamma}_{1}$ has full rank too, and consequently, that the cost functional is also conditionally continuous on $P_{a}$, which is tantamount to the law of one price. A 
conditional version of the Riesz representation theorem then implies that there exist two unique elements of $P_{a}$ that represent these conditional functionals over $P_{a} \cdot{ }^{6}$ In particular, the active mean and cost representing portfolios, $p_{a}^{+}$and $p_{a}^{*}$, respectively, will be such that:

$$
E\left(p \mid G_{1}\right)=E\left(p_{a}^{+} p \mid G_{1}\right), \quad C\left(p \mid G_{1}\right)=E\left(p_{a}^{*} p \mid G_{1}\right), \quad \forall p \in P_{a}
$$

It is then straightforward to show that

$$
p_{a}^{+}=\mathbf{x}^{\prime} \boldsymbol{\Gamma}_{\mathbf{1}}^{-1} \boldsymbol{\nu}_{\mathbf{1}}, \quad p_{a}^{*}=\mathbf{x}^{\prime} \boldsymbol{\Gamma}_{\mathbf{1}}^{-1} \mathbf{c}_{\mathbf{1}} .
$$

If $P_{a}$ included the conditionally (and unconditionally) safe payoff $x_{0}=1$, then $p_{a}^{+}$would coincide with it. But even though it does not, it follows from (3) that we can interpret $p_{a}^{+}$as the conditional projection of $x_{0}$ onto $P_{a}$. We can also use (3) to interpret $p_{a}^{*}$ as the conditional projection of any valid SDF onto $P_{a}$. As is well known, a SDF is any scalar random variable $m \in I_{2}$ that prices all conceivable payoffs in terms of their expected cross product with it. More formally,

$$
E\left(m p \mid G_{1}\right)=C\left(p \mid G_{1}\right), \quad \forall p \in P_{a} .
$$

Equivalently, admissible SDFs are fully characterised by the condition

$$
E\left(m \mathbf{x} \mid G_{1}\right)=\mathbf{c}_{1}
$$

since they satisfy $E\left[\mathbf{w}_{1}^{\prime}\left(m \mathbf{x}-\mathbf{c}_{1}\right) \mid G_{1}\right]=0$ for any $\mathbf{w}_{1} \in I_{1}$. In addition, since $C\left(1 \mid G_{1}\right)=$ $E\left(1 \cdot m \mid G_{1}\right)$, the conditionally expected value of $m$ defines the shadow price of the unit payoff. In practice, each (frictionless) asset pricing model can be represented by a particular SDF. For instance, the CAPM states that $m$ is affine in the return of the market portfolio, while the CCAPM implies that $m$ is the intertemporal marginal rate of substitution in consumption of the representative agent.

Finally, it is worth mentioning that both representing portfolios and SDFs can also be defined in terms of unconditional moments. Specifically, the law of iterated expectations implies that $p_{a}^{+}$and $p_{a}^{*}$ also represent unconditional means and average costs on the active payoff space $P_{a}$, so that:

$$
E(p)=E\left(p_{a}^{+} p\right), \quad C(p)=E\left(p_{a}^{*} p\right), \quad \forall p \in P_{a} .
$$

\footnotetext{
${ }^{6}$ Chamberlain and Rothschild (1983) introduced mean and cost representing portfolios to study unconditional mean-variance analysis in infinite dimensional payoff spaces in which conditional information plays no role. Hansen and Richard (1987) extended their results to conditioning information.
} 
Similarly, we could also define SDFs as those $m \in I_{2}$ that give the right pseudo-price for any conceivable $p$, i.e.

$$
E(m p)=C(p), \quad \forall p \in P_{a}
$$

Therefore, as Hansen and Richard (1987) point out, there is no loss of information in moving from pricing to pseudo-pricing, but only as long as we focus on the whole of $P_{a}$, and not simply on a subset, as in sections 5 and 6 .

\section{Conditional Mean-Variance Frontiers}

Let us begin by focusing on the first column of Table 1, that is, those active portfolio strategies that are optimal with respect to conditional moments. These frontiers are the relevant ones for both informed investors and researchers, even though it is difficult to construct them in practice, as they require the correct specification of the first two moments of the joint distribution of asset returns given the agents' information set.

\subsection{Conditional Return and SDF Frontiers: CRF and CSF}

Hansen and Richard (1987) define the Conditional Return Mean-Variance Frontier (CRF) as the highest lower bound on conditional variances for a given profile of conditional expected returns that can be achieved by portfolios whose weights may depend on conditioning information, but whose price is always one. Thus, the CRF will be given by the set of active portfolio strategies that solve the non-standard optimisation problem

$$
\min _{p \in P_{a}} E\left(p^{2} \mid G_{1}\right) \quad \text { s.t. } \quad E\left(p \mid G_{1}\right)=\bar{\nu}_{1}, \quad C\left(p \mid G_{1}\right)=1
$$

where, importantly, the objective function is a random variable in $I_{1}$, while the first constraint imposes a particular conditional mean profile on the returns that can be considered. Hansen and Richard (1987) go on to show that the active portfolio strategies that solve (6) can be represented as

$$
\begin{aligned}
& p_{C}\left(\bar{\nu}_{1}\right)=R_{a}^{*}+\omega_{1}\left(\bar{\nu}_{1}\right) A_{a}^{+}, \\
& \omega_{1}\left(\bar{\nu}_{1}\right)=\frac{\bar{\nu}_{1}-\mathrm{D}_{1}}{\mathrm{E}_{1}},
\end{aligned}
$$

where

$$
R_{a}^{*}=\frac{1}{\mathrm{C}_{1}} p_{a}^{*}
$$


is the return associated to the active cost representing portfolio,

$$
A_{a}^{+}=p_{a}^{+}-\frac{\mathrm{A}_{1}}{\mathrm{C}_{1}} p_{a}^{*}
$$

is the residual from the conditional projection of $p_{a}^{+}$onto $\left\langle p_{a}^{*}\right\rangle_{1},{ }^{7}$ and

$$
\begin{gathered}
\mathrm{A}_{1}=\boldsymbol{\nu}_{1}^{\prime} \boldsymbol{\Gamma}_{1}^{-1} \mathbf{c}_{1}=E\left(p_{a}^{*} \mid G_{1}\right)=C\left(p_{a}^{+} \mid G_{1}\right)=E\left(p_{a}^{+} p_{a}^{*} \mid G_{1}\right), \\
\mathrm{B}_{1}=\boldsymbol{\nu}_{1}^{\prime} \boldsymbol{\Gamma}_{1}^{-1} \boldsymbol{\nu}_{1}=E\left(p_{a}^{+} \mid G_{1}\right)=E\left(p_{a}^{+2} \mid G_{1}\right), \\
\mathrm{C}_{1}=\mathbf{c}_{1}^{\prime} \boldsymbol{\Gamma}_{1}^{-1} \mathbf{c}_{1}=C\left(p_{a}^{*} \mid G_{1}\right)=E\left(p_{a}^{* 2} \mid G_{1}\right), \\
\mathrm{D}_{1}=\mathrm{A}_{1} / \mathrm{C}_{1}=E\left(R_{a}^{*} \mid G_{1}\right), \\
\mathrm{E}_{1}=\mathrm{B}_{1}-\mathrm{A}_{1}^{2} / \mathrm{C}_{1}=E\left(A_{a}^{+} \mid G_{1}\right)=E\left(A_{a}^{+2} \mid G_{1}\right),
\end{gathered}
$$

all of which belong to $I_{1}$. Finally, Hansen and Richard (1987) also mention that there is conditional two-fund spanning on the CRF, so that any element on the CRF can be replicated by an active portfolio of two other elements on the CRF.

In this context, the conditional second moment of portfolios on the CRF is given by

$$
E\left[p_{C}^{2}\left(\bar{\nu}_{1}\right) \mid G_{1}\right]=\mathrm{F}_{1}+\omega_{1}^{2}\left(\bar{\nu}_{1}\right) \mathrm{E}_{1},
$$

where

$$
\mathrm{F}_{1}=\frac{1}{\mathrm{C}_{1}}=E\left(R_{a}^{* 2} \mid G_{1}\right) .
$$

As a consequence, the CRF will be a parabola in $\left[\operatorname{Var}\left(p \mid G_{1}\right), E\left(p \mid G_{1}\right)\right]$ space and a hyperbola in $\left[\sqrt{\operatorname{Var}\left(p \mid G_{1}\right)}, E\left(p \mid G_{1}\right)\right]$ space for a particular value of the conditioning variables in $G_{1}$.
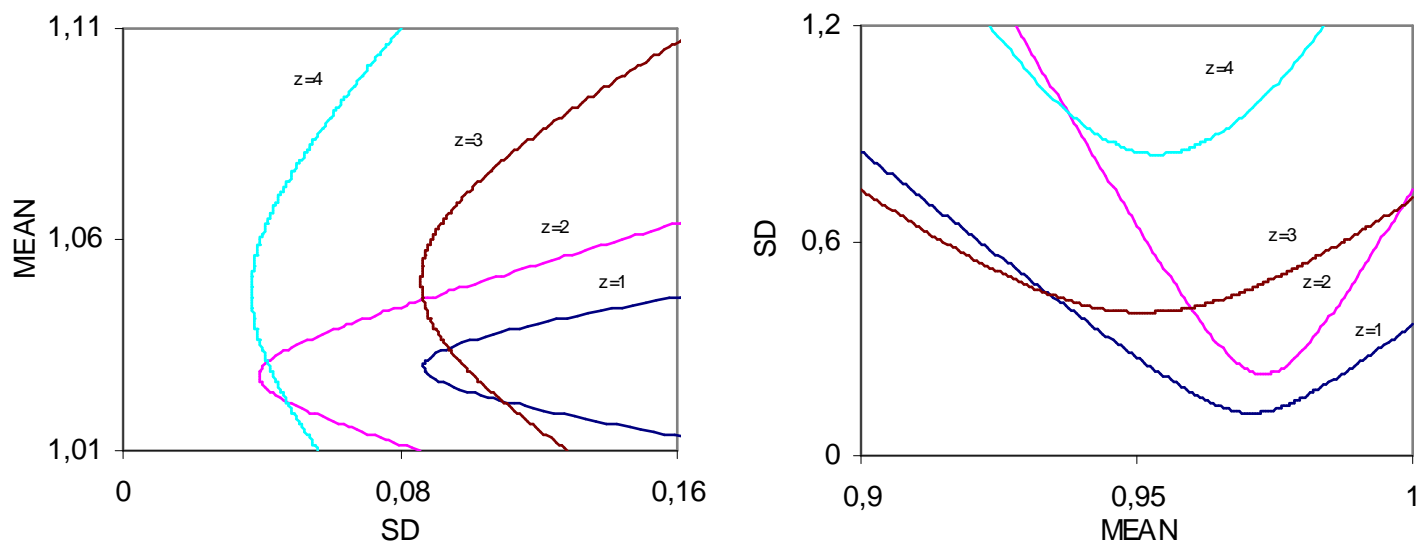

Figure 1: Mean-variance frontiers conditional on specific signal values. Portfolio frontiers on the left and SDF frontiers on the right.

\footnotetext{
${ }^{7} A_{a}^{+}$can be interpreted as the mean representing portfolio in the space of arbitrage portfolios, i.e. the unique arbitrage portfolio that satisfies $E\left(A_{a}^{+} p \mid G_{1}\right)=E\left(p \mid G_{1}\right)$ for any portfolio $p \in P_{a}$ such that $C\left(p \mid G_{1}\right)=0$.
} 
The first panel of Figure 1 illustrates the CRF for the multinomial illustration described in section 2.2. Given a specific mean profile $\bar{\nu}_{1}\left(=\bar{\nu}_{1}^{1}, \bar{\nu}_{1}^{2}, \bar{\nu}_{1}^{3}, \bar{\nu}_{1}^{4}\right)$, the position of the corresponding $p_{C}\left(\bar{\nu}_{1}\right)$ in $(7)$ can be easily located.

On the other hand, Gallant, Hansen and Tauchen (1990) define the Conditional SDF MeanVariance Frontier (CSF) as the highest lower bound on the conditional variance of those SDFs that correctly price all the active portfolios that can be generated from the vector of asset payoffs $\mathbf{x}$, as defined in (5). Hence, the CSF will be given by the set of scalar random variables that solve the non-standard optimisation problem

$$
\min _{m \in I_{2}} E\left(m^{2} \mid G_{1}\right) \quad \text { s.t. } \quad E\left(m \mid G_{1}\right)=\bar{c}_{1}, \quad E\left(m \mathbf{x} \mid G_{1}\right)=\mathbf{c}_{1},
$$

where, once again, the objective function is a random variable in $I_{1}$, while the first constraint imposes a particular mean profile on the potential SDFs that can be considered. Gallant, Hansen and Tauchen (1990) go on to show that the solution to (10) can be represented as

$$
\begin{aligned}
m_{C}\left(\bar{c}_{1}\right) & =p_{a}^{*}+\varpi_{1}\left(\bar{c}_{1}\right) E_{a}^{+}, \\
\varpi_{1}\left(\bar{c}_{1}\right) & =\frac{\bar{c}_{1}-\mathrm{A}_{1}}{1-\mathrm{B}_{1}},
\end{aligned}
$$

where $p_{a}^{*}$ was defined in (4) and

$$
E_{a}^{+}=1-p_{a}^{+}
$$

is the residual from the conditional projection of 1 onto $\langle\mathbf{x}\rangle_{1}$. Finally, it is also possible to show that there is conditional two fund spanning on the CSF, in the sense that we can use a conditionally linear combination of two elements of the CSF to replicate any other element.

In this context, the conditional second moment of SDFs on the CSF is given by

$$
E\left[m_{C}^{2}\left(\bar{c}_{1}\right) \mid G_{1}\right]=\mathrm{C}_{1}+\varpi_{1}^{2}\left(\bar{c}_{1}\right)\left(1-\mathrm{B}_{1}\right) .
$$

Therefore, the CSF will also be a parabola in $\left[E\left(m \mid G_{1}\right), \operatorname{Var}\left(m \mid G_{1}\right)\right]$ space and a hyperbola in $\left[E\left(m \mid G_{1}\right), \sqrt{\operatorname{Var}\left(m \mid G_{1}\right)}\right]$ space for a given value of the conditioning variables in $G_{1}$. The second panel of Figure 1 illustrates the CSF for the multinomial illustration described in section 2.2. Given a specific mean profile $\bar{c}_{1}\left(=\bar{c}_{1}^{1}, \bar{c}_{1}^{2}, \bar{c}_{1}^{3}, \bar{c}_{1}^{4}\right)$, the position of the corresponding $m_{C}\left(\bar{c}_{1}\right)$ in (11) can be easily located. 


\subsection{Duality between CSF and CRF}

Expression (11) implies that the CSF can be represented as the sum of two components: $p_{a}^{*}-\varpi_{1}\left(\bar{c}_{1}\right) p_{a}^{+}$, which belongs to $P_{a}$ and can therefore be traded, and $\varpi_{1}\left(\bar{c}_{1}\right)$, which cannot be traded in the absence of a conditionally safe asset. In this context, the following question arises: Is the traded component of $m_{C}\left(\bar{c}_{1}\right)$ related to the CRF? A simple example is $p_{a}^{*}$, which belongs to the CSF, while its return $R_{a}^{*}$ belongs to the CRF. The following proposition, which relates the mean profile of a given element of one frontier to the mean profile of some element of the other frontier for every signal value, extends to active strategies the well-known duality results obtained by Hansen and Jagannathan (1991) for passive strategies (see section 6.2 below):

Proposition 1 Let $\bar{\nu}_{1}$ and $\bar{c}_{1}$ denote some specific conditional mean profiles for the CRF and $C S F$, respectively, and let $\omega_{1}\left(\bar{\nu}_{1}\right)$ and $\varpi_{1}\left(\bar{c}_{1}\right)$ be the conditional weights on $A_{a}^{+}$and $E_{a}^{+}$of the corresponding CRF and CSF elements (7) and (11), respectively. Then:

1. The traded component of any element of the CSF $m_{C}\left(\bar{c}_{1}\right)$ such that $\mathrm{C}_{1}-\varpi_{1}\left(\bar{c}_{1}\right) \mathrm{A}_{1} \neq 0$ will be conditionally proportional to some element of the CRF $p_{C}\left(\bar{\nu}_{1}\right)$ if and only if the conditional mean profiles $\bar{\nu}_{1}$ and $\bar{c}_{1}$ satisfy

$$
\varpi_{1}\left(\bar{c}_{1}\right)-\mathrm{A}_{1} \varpi_{1}\left(\bar{c}_{1}\right) \omega_{1}\left(\bar{\nu}_{1}\right)+\mathrm{C}_{1} \omega_{1}\left(\bar{\nu}_{1}\right)=0 .
$$

2. Any element of the CRF $p_{C}\left(\bar{\nu}_{1}\right)$ such that $1-\omega_{1}\left(\bar{\nu}_{1}\right) \mathrm{A}_{1} \neq 0$ will be conditionally proportional to the traded component of some element of the CSF $m_{C}\left(\bar{c}_{1}\right)$ if and only if the profiles $\bar{\nu}_{1}$ and $\bar{c}_{1}$ satisfy (13).

As a consequence, there is an element-by-element duality between the CRF and CSF frontiers, in the sense that given an element of one frontier, we can find its counterpart in the other one by choosing the conditional mean profile $\bar{\nu}_{1}$ or $\bar{c}_{1}$ in such a way that (13) is satisfied. Given that the relationship between those two dual elements is conditionally affine, they will show perfect conditional correlation. The first panel of Figure 2 illustrates this duality for a particular value of the signals.

Strictly speaking, there are two exceptions to this rule. Still, in both cases we can establish a link between an element of one frontier and the asymptotes of the other. More specifically, the exception to the first part of Proposition 1 occurs when the conditional mean profile $\bar{c}_{1}$ is such that $\mathrm{C}_{1}-\varpi_{1}\left(\bar{c}_{1}\right) \mathrm{A}_{1}=0$. In that case,

$$
m_{C}\left[\frac{1}{\mathrm{D}_{1}}\left(1-\mathrm{E}_{1}\right)\right]=\frac{1}{\mathrm{D}_{1}}\left(1-A_{a}^{+}\right),
$$

which does not have a position on $R^{*}$ as required by the CRF. Intuitively, we need the cost of the traded element of $m_{C}\left(\bar{c}_{1}\right)$ to be different from zero for every possible realisation of the 
signals in order to be able to construct a return. However, as we let $\left|\bar{\nu}_{1}\right|$ grow without bound, the term $A_{a}^{+}$becomes the main driver of $p_{C}\left(\bar{\nu}_{1}\right)$, in the sense that

$$
\lim _{\bar{\nu}_{1} \rightarrow \pm \infty} E\left[\left(\frac{p_{C}\left(\bar{\nu}_{1}\right)}{\bar{\nu}_{1}}-\frac{A_{a}^{+}}{\mathrm{E}_{1}}\right)^{2} \mid G_{1}\right]=\lim _{\bar{\nu}_{1} \rightarrow \pm \infty} \frac{1}{\bar{\nu}_{1}^{2}} E\left[\left(R_{a}^{*}-\frac{\mathrm{D}_{1}}{\mathrm{E}_{1}} A_{a}^{+}\right)^{2} \mid G_{1}\right]=0,
$$

and we can only relate $m_{C}\left[\left(1-\mathrm{E}_{1}\right) / \mathrm{D}_{1}\right]$ to the asymptotes of the CRF

$$
\lim _{\bar{\nu}_{1} \rightarrow \pm \infty} \frac{\sqrt{\operatorname{Var}\left[p_{C}\left(\bar{\nu}_{1}\right) \mid G_{1}\right]}}{\bar{\nu}_{1}}= \pm \sqrt{\frac{1-\mathrm{E}_{1}}{\mathrm{E}_{1}}} .
$$
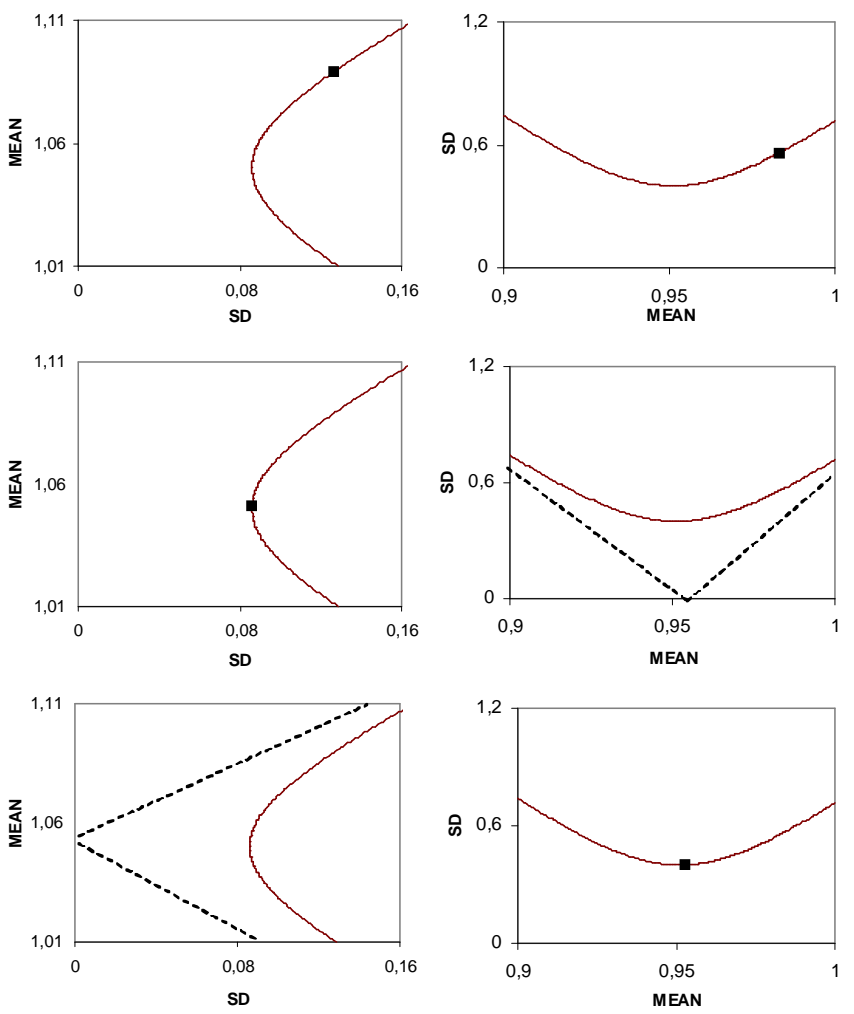

Figure 2: Duality between conditional frontiers and its exceptions at a particular signal value.

Portfolio frontiers on the left and SDF frontiers on the right.

Similarly, the exception to the second part of Proposition 1 occurs when the conditional mean profile $\bar{\nu}_{1}$ is such that $1-\omega_{1}\left(\bar{\nu}_{1}\right) \mathrm{A}_{1}=0$. In that case,

$$
p_{C}\left(\frac{\mathrm{B}_{1}}{\mathrm{~A}_{1}}\right)=\frac{1}{\mathrm{~A}_{1}} p_{a}^{+}
$$

which does not have a position on $p_{a}^{*}$ as required by the CSF. However, as we let $\left|\bar{c}_{1}\right|$ grow without bound, the term $E_{a}^{+}$becomes the main driver of $m^{C}\left(\bar{c}_{1}\right)$, in the sense that

$$
\lim _{\bar{c}_{1} \rightarrow \pm \infty} E\left[\left(\frac{m_{C}\left(\bar{c}_{1}\right)}{\bar{c}_{1}}-\frac{E_{a}^{+}}{1-\mathrm{B}_{1}}\right)^{2} \mid G_{1}\right]=\lim _{\bar{c}_{1} \rightarrow \pm \infty} \frac{1}{\bar{c}_{1}^{2}} E\left[\left(p_{a}^{*}-\frac{\mathrm{A}_{1}}{1-\mathrm{B}_{1}} E_{a}^{+}\right)^{2} \mid G_{1}\right]=0
$$


and we can relate $p_{C}\left(\mathrm{~B}_{1} / \mathrm{A}_{1}\right)$ to the asymptotes of the CSF

$$
\lim _{\bar{c}_{1} \rightarrow \pm \infty} \frac{\sqrt{\operatorname{Var}\left[m_{C}\left(\bar{c}_{1}\right) \mid G_{1}\right]}}{\bar{c}_{1}}= \pm \sqrt{\frac{\mathrm{B}_{1}}{1-\mathrm{B}_{1}}} .
$$

The two duality exceptions are illustrated for a particular signal value in the second and third panels of Figure 2, respectively.

\section{Unconditional Mean-Variance Frontiers}

Let us now focus on those active portfolio strategies that are optimal with respect to unconditional moments, which correspond to columns 2 and 3 in Table 1. At first sight, it might seem odd to study unconditional moments when we think of active strategies whose weights depend on conditioning information. However, in many practical situations the observer of the agents' decisions only has access to an information set that is much coarser than the agents' information set. The performance evaluation of a portfolio manager by means of the first two unconditional moments of her returns is a typical example of the use of unconditional return frontiers by an outside evaluator who may not have access to the proprietary strategies followed by the manager. Similarly, the evaluation of a specific asset pricing model by computing the first and second unconditional moments of the corresponding SDF is the typical example of the use of unconditional SDF frontiers by an econometrician who wants to avoid the use of the wrong conditional model for returns.

\subsection{Unconditional Return and SDF frontiers: URF and USF}

Hansen and Richard (1987) define the Unconditional Return Mean-Variance Frontier (URF) as the highest lower bound on the variance for each level of expected return that can be achieved by portfolios with weights that may depend on conditioning information, but whose price is always one. Hence, the URF will be given by the set of active portfolio strategies that solve the more standard problem

$$
\min _{p \in P_{a}} E\left(p^{2}\right) \quad \text { s.t. } \quad E(p)=\bar{\nu}, \quad C\left(p \mid G_{1}\right)=1,
$$

where both the objective function and the mean constraint are now real-valued. Hansen and Richard (1987) go on to show that the gross returns that solve (14) can be represented as

$$
\begin{aligned}
p_{U}(\bar{\nu}) & =R_{a}^{*}+\omega_{U}(\bar{\nu}) A_{a}^{+}, \\
\omega_{U}(\bar{\nu}) & =\frac{\bar{\nu}-E\left(\mathrm{D}_{\mathbf{1}}\right)}{E\left(\mathrm{E}_{\mathbf{1}}\right)},
\end{aligned}
$$


where $R_{a}^{*}$ and $A_{a}^{+}$are defined in (8) and (9), respectively. Importantly, note that in contrast to $(7)$, the weight on $A_{a}^{+}$is no longer conditional on $G_{1} \cdot{ }^{8} \quad$ Further, it directly follows from the results in Hansen and Richard (1987) that there is unconditional two fund spanning on the URF, in the sense that a passive strategy of two elements of the URF can replicate any other element.

In this context, the unconditional second moment of portfolios on the URF will be given by

$$
E\left[p_{U}^{2}(\bar{\nu})\right]=E\left(\mathrm{~F}_{\mathbf{1}}\right)+\omega_{U}^{2}(\bar{\nu}) E\left(\mathrm{E}_{\mathbf{1}}\right)
$$

As a consequence, the URF will be a hyperbola in $[\sqrt{\operatorname{Var}(p)}, E(p)]$ space.

On the other hand, Gallant, Hansen and Tauchen (1990) define the Unconditional SDF Mean-Variance Frontier (USF) as the highest lower bound on the unconditional variance of those SDFs that correctly price all the active portfolios that can be generated from the vector of asset payoffs $\mathbf{x}$, as defined in (5). Hence, the USF will be given by the set of scalar random variables that solve the more standard optimisation problem

$$
\min _{m \in I_{2}} E\left(m^{2}\right) \quad \text { s.t. } \quad E(m)=\bar{c}, \quad E\left(m \mathbf{x} \mid G_{1}\right)=\mathbf{c}_{1},
$$

where both the objective function and the first constraint are now real-valued. Gallant, Hansen and Tauchen (1990) go on to show that the solution to (16) can be represented as

$$
\begin{aligned}
m_{U}(\bar{c}) & =p_{a}^{*}+\varpi_{U}(\bar{c}) E_{a}^{+}, \\
\varpi_{U}(\bar{c}) & =\frac{\bar{c}-E\left(\mathrm{~A}_{1}\right)}{1-E\left(\mathrm{~B}_{1}\right)},
\end{aligned}
$$

where $p_{a}^{*}$ and $E_{a}^{+}$are defined in (4) and (12), respectively. Importantly, note that in contrast to (11), the weight on $E_{a}^{+}$is no longer conditional on $G_{1} \cdot{ }^{9} \quad$ One can also show that there is unconditional two fund spanning on the USF, in the sense that we can use an unconditional combination of two elements on $m_{U}(\bar{c})$ to replicate any other element on $m_{U}(\bar{c})$.

\footnotetext{
${ }^{8}$ Perhaps the best known result of Hansen and Richard (1987) is that while unconditional frontier portfolios always lie on the conditional frontier, the converse is not generally true. More specifically, a CRF portfolio will also be located on the URF if and only if we choose the conditional mean profile as

$$
\bar{\nu}_{1}=\left[\frac{\bar{\nu}-E\left(\mathrm{D}_{1}\right)}{E\left(\mathrm{E}_{1}\right)}\right] \mathrm{E}_{1}+\mathrm{D}_{1},
$$

so that $\omega_{1}\left(\bar{\nu}_{1}\right)$ is in fact constant $\left(=\omega_{U}(\bar{\nu})\right)$.

${ }^{9}$ Another important result of Gallant et al. (1990) is that while unconditional frontier SDFs always lie on the conditional frontier, the converse is not generally true. More specifically, a SDF on the CSF will also be located on the USF if and only if we choose the conditional mean profile as

$$
\bar{c}_{1}=\left[\frac{\bar{c}-E\left(\mathrm{~A}_{1}\right)}{1-E\left(\mathrm{~B}_{1}\right)}\right]\left(1-\mathrm{B}_{1}\right)+\mathrm{A}_{1},
$$

so that $\varpi_{1}\left(\bar{c}_{1}\right)$ is in fact constant $\left(=\varpi_{U}(\bar{c})\right)$.
} 
Finally, the unconditional second moment of SDFs on the USF is given by

$$
E\left[m_{U}^{2}(\bar{c})\right]=E\left(\mathrm{C}_{\mathbf{1}}\right)+\varpi_{U}^{2}(\bar{c})\left(1-E\left(\mathrm{~B}_{\mathbf{1}}\right)\right)
$$

Therefore, the USF will also be a hyperbola in $[E(m), \sqrt{\operatorname{Var}(m)}]$ space. Figure 3 illustrates the shapes that the URF and USF can take for the multinomial illustration described in section 2.2 .
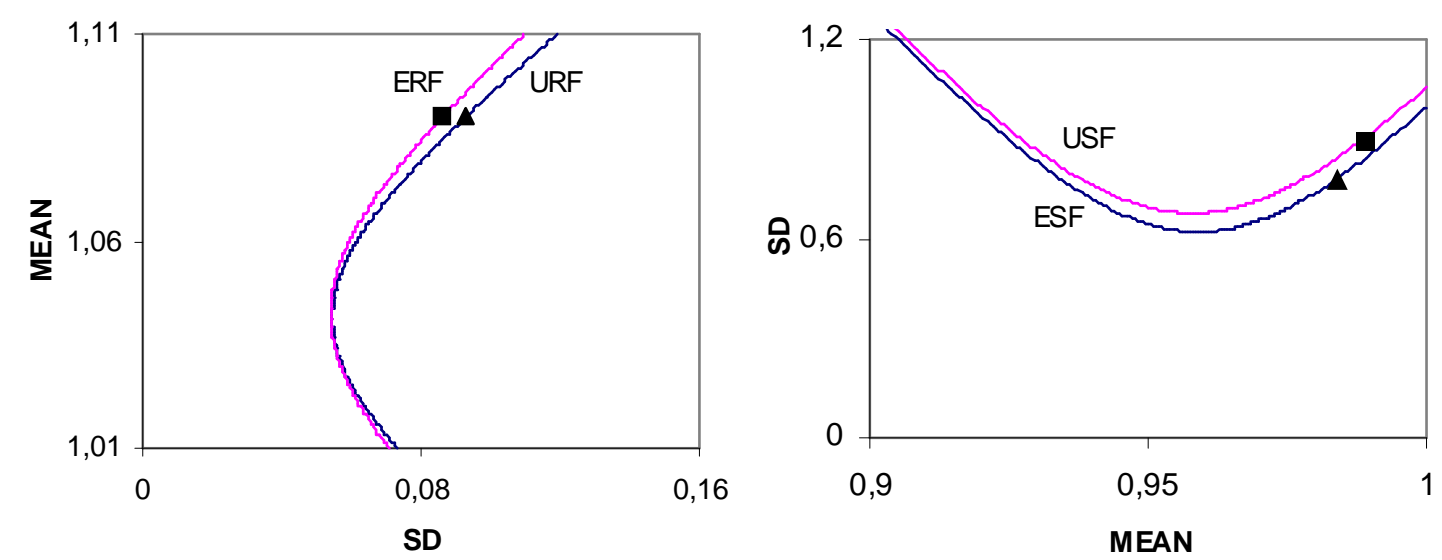

Figure 3: Unconditional and extended frontiers and their duality. Portfolio frontiers on the left and SDF frontiers on the right.

\subsection{Lack of Duality between USF and URF}

Given the close analogies between conditional and unconditional return and SDF frontiers, one is tempted to conclude that there must exist an unconditional analogue to Proposition 1. Moreover, the fact that $p_{a}^{*}$ belongs to the USF, while its return $R_{a}^{*}$ belongs to the URF strengthens such an intuition. As our next result shows, however, it turns out that $p_{a}^{*}$ and $R_{a}^{*}$ are the only dual points:

Proposition 2 Let $\bar{\nu}$ and $\bar{c}$ denote some specific means for the URF and USF, respectively, and let $\omega_{U}(\bar{\nu})$ and $\varpi_{U}(\bar{c})$ be the unconditional weights on $A_{a}^{+}$and $E_{a}^{+}$of the corresponding URF and USF elements (15) and (17), respectively. Then:

1. The traded component of any element of the USF $m_{U}(\bar{c})$ such that $\mathrm{C}_{\mathbf{1}}-\varpi_{U}(\bar{c}) \mathrm{A}_{\mathbf{1}} \neq 0$ is conditionally proportional to some element of the URF $p_{U}(\bar{\nu})$ if and only if $\bar{\nu}$ and $\bar{c}$ satisfy

$$
\varpi_{U}(\bar{c})-\mathrm{A}_{1} \omega_{U}(\bar{\nu}) \varpi_{U}(\bar{c})-\mathrm{C}_{1} \omega_{U}(\bar{\nu})=0 .
$$

2. Any element of the URF $p_{U}(\bar{\nu})$ such that $1-\omega_{U}(\bar{\nu}) \mathrm{A}_{1} \neq 0$ is conditionally proportional to the traded part of some element of $m_{U}(\bar{c})$ if and only if $\bar{\nu}$ and $\bar{c}$ satisfy (18). 
Condition (18) trivially holds if we choose $\bar{\nu}=E\left(\mathrm{D}_{1}\right)$ and $\bar{c}=E\left(\mathrm{~A}_{1}\right)$, so that $\omega_{U}\left[E\left(\mathrm{D}_{1}\right)\right]=$ $\varpi_{U}\left[E\left(\mathrm{~A}_{1}\right)\right]=0$, which confirms the unconditional dual character of $R_{a}^{*}$ and $p_{a}^{*}$. But in general, it is not possible to find any other real numbers $(\bar{\nu}, \bar{c})$ such that the stochastic left hand side of (18) is 0 for every conceivable realisation of the signals in $G_{1}$. For this reason, the next section is devoted to the dual objects to the URF and USF. More specifically, we will characterise in full the random variables for which the dual objects to these two unconditional frontiers are themselves frontiers, as opposed to mere bounds.

\section{$5 \quad$ Extended Mean-Variance Frontiers}

Let us again study optimal active strategies from the point of view of unconditional moments in columns 2 and 3 of Table 1 , but this time with weaker pricing constraints. In particular, we will consider a portfolio frontier whose elements are not proper returns and a SDF frontier whose elements only price constant cost portfolios on average.

\subsection{Extended Return Frontier: ERF}

\subsubsection{Extended Returns and Extended Arbitrage Portfolios}

We define extended returns as portfolios with unitary average cost,${ }^{10}$ so that

$$
C(p)=E\left[C\left(p \mid G_{1}\right)\right]=1 .
$$

Obviously, one could transform an extended return into a proper return by dividing its weights by $C\left(p \mid G_{1}\right)$ if $C\left(p \mid G_{1}\right) \neq 0$, but in general such a transformation would depend on $G_{1}$.

In our multinomial illustration described in section 2.2 , extended returns satisfy

$$
C(p)=\sum_{k=1}^{4} \pi^{k} \mathbf{w}_{\perp}^{k \prime} \mathbf{c}_{\perp}^{k}=1
$$

while returns satisfy the stronger condition

$$
C(p \mid z=k)=\mathbf{w}_{1}^{k \prime} \mathbf{c}_{1}^{k}=1, \quad k=1,2,3,4 .
$$

Similarly, we can also define extended arbitrage portfolios as

$$
C(p)=E\left[C\left(p \mid G_{1}\right)\right]=0 .
$$

\footnotetext{
${ }^{10}$ Hansen and Richard (1987) refer to them as pseudo-returns.
} 


\subsubsection{Mean-Variance Frontier for Extended Returns}

By analogy with the frontiers discussed in the previous section, we define the Extended Return Mean-Variance Frontier (ERF) as the highest lower bound on the variance for each level of expected return that can be achieved by portfolios with weights that may depend on conditioning information, but whose price is only one on average. More formally, the ERF is the set of active portfolio strategies that solve the problem

$$
\min _{p \in P_{a}} E\left(p^{2}\right) \quad \text { s.t. } \quad E(p)=\bar{\nu}, \quad C(p)=1,
$$

which is an unconditional mean-variance problem similar to (14), but in the space of extended returns. Therefore, it is perhaps not surprising that as in the case of the URF, we can represent its solution by the following orthogonal decomposition:

Proposition 3 The solution to program (19) is given by

$$
\begin{aligned}
p_{E}(\bar{\nu}) & =R_{e}^{*}+\omega_{E}(\bar{\nu}) A_{e}^{+}=\left[\frac{\mathrm{C}_{1}}{E\left(\mathrm{C}_{1}\right)}+\omega_{E}(\bar{\nu})\left(\mathrm{A}_{1}-\frac{\mathrm{C}_{1} E\left(\mathrm{~A}_{1}\right)}{E\left(\mathrm{C}_{1}\right)}\right)\right] R_{a}^{*}+\omega_{E}(\bar{\nu}) A_{a}^{+}, \\
\omega_{E}(\bar{\nu}) & =\frac{\bar{\nu}-E\left(\mathrm{~A}_{1}\right) / E\left(\mathrm{C}_{1}\right)}{E\left(\mathrm{~B}_{1}\right)-E^{2}\left(\mathrm{~A}_{1}\right) / E\left(\mathrm{C}_{1}\right)}
\end{aligned}
$$

where

$$
R_{e}^{*}=\frac{1}{E\left(\mathrm{C}_{1}\right)} p_{a}^{*}
$$

is the extended return associated to the cost representing portfolio, and

$$
A_{e}^{+}=p_{a}^{+}-\frac{E\left(\mathrm{~A}_{1}\right)}{E\left(\mathrm{C}_{1}\right)} p_{a}^{*}
$$

which is the residual from the unconditional projection of $p_{a}^{+}$onto $\left\langle p_{a}^{*}\right\rangle$, is the (unconditional) mean representing portfolio is the space of extended arbitrage portfolios.

The main difference between expressions (15) and (20) is that in the latter the weight on $R_{a}^{*}$ is not systematically one, although it is one on average. ${ }^{11}$ We can also show that there is unconditional two fund spanning on the ERF, in the sense that a passive strategy of two elements of the ERF can replicate any other element.

Finally, given that the second moment of the portfolios on the ERF is

$$
E\left[p_{E}^{2}(\bar{\nu})\right]=\frac{1}{E\left(\mathrm{C}_{\mathbf{1}}\right)}+\omega_{E}^{2}(\bar{\nu})\left[E\left(\mathrm{~B}_{\mathbf{1}}\right)-\frac{E^{2}\left(\mathrm{~A}_{\mathbf{1}}\right)}{E\left(\mathrm{C}_{\mathbf{1}}\right)}\right]
$$

the ERF will also be a hyperbola in $[\sqrt{\operatorname{Var}(p)}, E(p)]$ space. Figure 3 shows the ERF jointly with the URF. As this figure illustrates, the ERF will generally be to the left of the URF on

\footnotetext{
${ }^{11}$ Therefore, the elements of the ERF do not generally belong to the CRF, unlike the elements of the URF (see footnote 8). In this sense, note that in general $R_{a}^{*}$ will not belong to the ERF, while $R_{e}^{*}$ always will.
} 
this space because (19) has the same objective function as (14) but with less demanding cost constraints. Nevertheless, the relative position of the ERF in mean-variance space does not really reflect an improvement of the investors' investment opportunities with respect to the URF. As we mentioned before, the reason is that the conditional cost of the portfolios on the ERF is not necessarily 1 for every possible value of the signals in $G_{1}$, as in the case of returns, but only 1 on average, which renders them useless for an investor with positive wealth.

\subsubsection{Duality between USF and ERF}

Given that both the ERF and USF have constant weights on $\left(p_{a}^{*}, p_{a}^{+}\right)$, we might expect the elements of the ERF and the USF to be linked by an unconditionally affine relationship, and as a consequence to show perfect unconditional correlation. The following result characterises precisely the element-by-element duality between these two frontiers:

Proposition 4 Let $\bar{\nu}$ and $\bar{c}$ denote some specific means for the ERF and USF, respectively, and let $\omega_{E}(\bar{\nu})$ and $\varpi_{U}(\bar{c})$ be the unconditional weights on $A_{e}^{+}$and $E_{a}^{+}$of the corresponding ERF and USF elements (20) and (17), respectively. Then:

1. The traded component of any element of the $\operatorname{USF} m_{U}(\bar{c})$ such that $E\left(\mathrm{C}_{1}\right)-\varpi_{U}(\bar{c}) E\left(\mathrm{~A}_{1}\right) \neq$ 0 is unconditionally proportional to some element of the ERF $p_{E}(\bar{\nu})$ if and only if $\bar{\nu}$ and $\bar{c}$ satisfy

$$
\varpi_{U}(\bar{c})-E\left(\mathrm{~A}_{1}\right) \varpi_{U}(\bar{c}) \omega_{E}(\bar{\nu})-E\left(\mathrm{C}_{1}\right) \omega_{E}(\bar{\nu})=0 .
$$

2. Any element of the $E R F p_{E}(\bar{\nu})$ such that $1-\omega_{E}(\bar{\nu}) E\left(A_{1}\right) \neq 0$ is unconditionally proportional to the traded part of some element of the USF $m_{U}(\bar{c})$ if and only if $\bar{\nu}$ and $\bar{c}$ satisfy (23).

Figure 3 shows a particular dual point of the ERF and the USF. Note that while Proposition 2 showed that in general only one specific point on the URF (namely $R_{a}^{*}$ ) could be related to another specific point on the USF (namely $p_{a}^{*}$ ), Proposition 4 shows that there are only two elements on the ERF and the USF for which it is not possible to find a counterpart on the other frontier.

Those two duality exceptions are analogous to the ones in Proposition 1, and their geometry is analogous to Figure 2. The exception to the first part of Proposition 4 occurs when $\bar{c}$ is such that $E\left(\mathrm{C}_{\mathbf{1}}\right)-\varpi_{U}(\bar{c}) E\left(\mathrm{~A}_{1}\right)=0$, in which case the USF point is $E^{-1}\left(\mathrm{~A}_{\mathbf{1}}\right) E\left(\mathrm{C}_{\mathbf{1}}\right)\left(1-A_{e}^{+}\right)$, whose risky part has a zero average cost. Still, we can establish a link between this element of the USF and the asymptotes of the ERF, as it was the case of the CRF. Similarly, the exception to the second part of Proposition 4 occurs when $\bar{\nu}$ is such that $1-\omega_{E}(\bar{\nu}) E\left(\mathrm{~A}_{1}\right)=0$, in which case the ERF point is $E^{-1}\left(\mathrm{~A}_{1}\right) p_{a}^{+}$, which does not have any weight on $p_{a}^{*}$. But again, we can establish a 
link between this element of the ERF and the asymptotes of the USF, as it was the case of the CSF.

Finally, it is important to emphasise once again that although the USF delivers the optimal constraints on asset pricing models, its dual, i.e. the ERF, is useless from the vantage point of an investor.

\subsection{Extended SDF Frontier: ESF}

\subsubsection{Constant-Cost Payoff Space and Extended SDFs}

Let us now focus on constant conditional cost portfolios by defining the restricted payoff space $P_{e} \subset P_{a}$ as

$$
P_{e}=\left\{p \in P_{a}: C\left(p \mid G_{1}\right)=C(p)\right\},
$$

which includes returns and arbitrage portfolios. ${ }^{12}$

To clarify the constraint that a constant cost imposes on active strategies, it is convenient to re-express an arbitrary active strategy $p=\mathbf{x}^{\prime} \mathbf{w}_{\mathbf{1}}$ as

$$
p=R_{1} w_{1}+\left(\mathbf{x}_{-1}-R_{1} \mathbf{c}_{1,-1}\right)^{\prime} \mathbf{w}_{\mathbf{1},-1}
$$

where the subscript -1 means that we have deleted the first element of the corresponding vector, $R_{1}$ is the gross return on the first asset (which we have assumed that has a nonzero price without loss of generality), and the vector $\mathbf{x}_{-1}-R_{1} \mathbf{c}_{1,-1}$ transforms the remaining asset payoffs into arbitrage portfolios. In this way, we can establish a direct connection between the weight on $R_{1}$ and the portfolio cost because $C\left(p \mid G_{1}\right)=w_{\perp}$. Specifically, while the active payoff space $P_{a}$ does not impose any constraint on the dependence of $w_{1}$ and $\mathbf{w}_{1,-1}$ on the information in $G_{1}$, the constant-cost payoff space $P_{e}$ in contrast imposes the constraint $w_{1}=w \in \mathbb{R}$. Therefore, $p \in P_{e}$ if and only if

$$
p=R_{1} w+\left(\mathbf{x}_{-1}-R_{1} \mathbf{c}_{1,-1}\right)^{\prime} \mathbf{w}_{\mathbf{1},-1}, \quad w \in \mathbb{R}, \quad \mathbf{w}_{\mathbf{1},-1} \in I_{\mathbf{1}} .
$$

In this context, we define extended SDFs as those random variables $m \in I_{2}$ that price correctly on average any payoff that belongs to the constant-cost payoff space:

$$
E(m p)=C(p), \quad \forall p \in P_{e} .
$$

\footnotetext{
${ }^{12}$ Mathematically, $P_{e}$ has the structure of a subspace of $P_{a}$ with respect to unconditional linear combinations (passive strategies) of constant conditional cost portfolios.
} 
Given that (5) implies that proper SDFs satisfy an analogous condition for the richer set of payoffs in $P_{a}$, extended SDFs may not price correctly portfolios whose cost is not constant. The following lemma provides an equivalent characterisation for extended SDFs:

Lemma 1 Extended SDFs are fully characterised by the condition

$$
\begin{aligned}
E\left(m \mathbf{x} \mid G_{1}\right) & =h_{1} \mathbf{c}_{1}, \quad h_{1} \in I_{1} \\
E\left(h_{1}\right) & =1 .
\end{aligned}
$$

Obviously, any extended SDF could also be transformed into a true SDF by the normalisation $h_{1}^{-1} m$ if $h_{1} \neq 0$, but such a transformation would depend on $G_{1}$. In our multinomial illustration described in section 2.2, extended SDFs satisfy

$$
\begin{aligned}
E(m \mathbf{x} \mid z=k) & =h_{1}^{k} \mathbf{c}_{1}^{k}, \quad k=1,2,3,4, \\
\sum_{k=1}^{4} \pi^{k} h_{1}^{k} & =1
\end{aligned}
$$

while active SDFs must satisfy the stronger condition

$$
E(m \mathbf{x} \mid z=k)=\mathbf{c}_{1}^{k} \quad k=1,2,3,4 .
$$

\subsubsection{Mean-Variance Frontier for Extended SDFs}

The Extended SDFs Mean-Variance Frontier (ESF) yields the highest lower bound on the variance of those univariate random variables that price correctly on average any portfolio of $\mathbf{x}$ whose weights may depend on conditioning information, but whose cost is constant. Using Lemma 1, we can formally define the ESF as the set of scalar random variables $m$ that solve the problem

$$
\min _{m \in I_{2}} E\left(m^{2}\right) \quad \text { s.t. } \quad E(m)=\bar{c}, \quad E\left(m \mathbf{x} \mid G_{1}\right)=h_{1} \mathbf{c}_{1},
$$

where $h_{1} \in I_{1}$ is a scalar random variable such that $E\left(h_{1}\right)=1$. Given that $(25)$ is an unconditional mean-variance problem similar to (16), but in the space of extended SDFs, it is perhaps not surprising that we can also represent its solution by the following orthogonal decomposition:

Proposition 5 The solution to program (25) is given by

$$
\begin{aligned}
& m_{E}(\bar{c})=p_{e}^{*}+\varpi_{E}(\bar{c}) E_{e}^{+}=\left[\frac{\mathrm{F}_{1}}{E\left(\mathrm{~F}_{1}\right)}+\varpi_{E}(\bar{c})\left(\mathrm{D}_{1}-\frac{\mathrm{F}_{1} E\left(\mathrm{D}_{1}\right)}{E\left(\mathrm{~F}_{1}\right)}\right)\right] p_{a}^{*}+\varpi_{E}(\bar{c}) E_{a}^{+} \\
& \varpi_{E}(\bar{c})=\frac{\bar{c}-E\left(\mathrm{D}_{1}\right) / E\left(\mathrm{~F}_{1}\right)}{1-E\left(\mathrm{E}_{1}\right)-E^{2}\left(\mathrm{D}_{1}\right) / E\left(\mathrm{~F}_{1}\right)}
\end{aligned}
$$

where

$$
E_{e}^{+}=1-p_{e}^{+}
$$


and

$$
p_{e}^{*}=\frac{1}{E\left(\mathrm{~F}_{1}\right)} R_{a}^{*}, \quad p_{e}^{+}=A_{a}^{+}+\frac{E\left(\mathrm{D}_{1}\right)}{E\left(\mathrm{~F}_{1}\right)} R_{a}^{*},
$$

with $R_{a}^{*}$ and $A_{a}^{+}$defined in (8) and (9), respectively.

It is not difficult to prove that the portfolios $p_{e}^{*}$ and $p_{e}^{+}$are the two unique elements of $P_{e}$ that represent unconditional means and average costs on $P_{e}$. That is,

$$
E(p)=E\left(p_{e}^{+} p\right), \quad C(p)=E\left(p_{e}^{*} p\right), \quad \forall p \in P_{e}
$$

As a result, we will refer to $p_{e}^{+}$and $p_{e}^{*}$ as the extended mean and cost representing portfolios, respectively. Not surprisingly, $p_{e}^{+}$can be interpreted as the unconditional projection of a safe unit payoff $x_{0}$ onto $P_{e}, E_{e}^{+}$as its residual, and $p_{e}^{*}$ as the unconditional projection of extended SDFs onto $P_{e}$. Moreover, the return corresponding to $p_{e}^{*}$ is equal to $R_{a}^{*}$ (as opposed to $R_{e}^{*}$ in (21)), and the residual of the unconditional projection of $p_{e}^{+}$onto $\left\langle p_{e}^{*}\right\rangle$ is equal to $A_{a}^{+}$(as opposed to $A_{e}^{+}$in $\left.(22)\right)$.

The main difference between expressions (17) and (26) is that in the latter the weight on $p_{a}^{*}$ is not systematically one, only on average. ${ }^{13}$ We can also show that there is unconditional two fund spanning on the ESF, in the sense that an unconditionally linear combination of two elements of the ESF can replicate any other element.

Finally, given that the second moment of the extended SDFs on the ESF is

$$
E\left[m_{E}^{2}(\bar{c})\right]=\frac{1}{E\left(\mathrm{~F}_{\mathbf{1}}\right)}+\varpi_{E}^{2}(\bar{c})\left[1-E\left(\mathrm{E}_{\mathbf{1}}\right)-\frac{E^{2}\left(\mathrm{D}_{\mathbf{1}}\right)}{E\left(\mathrm{~F}_{\mathbf{1}}\right)}\right]
$$

the ESF will also be a hyperbola in $[E(m), \sqrt{\operatorname{Var}(m)}]$ space. Figure 3 shows the ESF jointly with the USF. As this figure illustrates, the ESF will be generally below the USF in this space because (25) has the same objective function as (16) but with less demanding pricing constraints. The elements of the USF price correctly on average every payoff in $P_{a}$, while those on the ESF do so for payoffs in $P_{e} \subset P_{a}$ only. As a result, we cannot usually guarantee that for a given $\bar{c}$ those optimal extended SDFs will provide the correct pricing over $P_{a}$.

\subsubsection{Duality Between ESF and URF}

Given that both the URF and ESF have constant weights on $\left(R_{a}^{*}, A_{a}^{+}\right)$, we might expect the elements of the URF and ESF to be linked by an unconditionally affine relationship, and as a

\footnotetext{
${ }^{13}$ Therefore, the elements of the ESF do not belong to the CSF, as it was the case for the elements of the USF. For instance, $p_{a}^{*}$ itself will not generally belong to the ESF, while $p_{e}^{*}$ always will.
} 
consequence, to show perfect unconditional correlation. The following result fully characterises the element-by-element duality between these two frontiers:

Proposition 6 Let $\bar{\nu}$ and $\bar{c}$ denote some specific means for the URF and ESF, respectively, and let $\omega_{U}(\bar{\nu})$ and $\varpi_{E}(\bar{c})$ be the unconditional weights on $A^{+}$and $E_{e}^{+}$of the corresponding URF and ESF elements (15) and (26), respectively. Then:

1. The traded component of any element of the ESF $m_{E}(\bar{c})$ such that $1-\varpi_{E}(\bar{c}) E\left(\mathrm{D}_{1}\right) \neq 0$ is unconditionally proportional to some element of the URF $p_{U}(\bar{v})$ if and only if $\bar{\nu}$ and $\bar{c}$ satisfy

$$
\varpi_{E}(\bar{c})-\frac{E\left(\mathrm{D}_{1}\right)}{E\left(\mathrm{~F}_{1}\right)} \varpi_{E}(\bar{c}) \omega_{U}(\bar{v})+\frac{1}{E\left(\mathrm{~F}_{1}\right)} \omega_{U}(\bar{v})=0 .
$$

2. Any element of the URF $p_{U}(\bar{v})$ such that $E\left(\mathrm{~F}_{1}\right)-\omega_{U}(\bar{v}) E\left(\mathrm{D}_{\mathbf{1}}\right) \neq 0$ is unconditionally proportional to the traded part of some element of the ESF $m_{E}(\bar{c})$ if and only if $\bar{\nu}$ and $\bar{c}$ satisfy (29).

Figure 3 shows a particular dual point of the ESF and the URF. Once again note that while Proposition 2 showed that in general only one specific point on the URF could be related to another specific point on the USF, Proposition 6 shows that there are only two elements on URF and ESF for which it is not possible to find a counterpart on the other frontier.

Those two duality exceptions are similar to the ones in Proposition 1 and their geometry is analogous to Figure 2. The exception to the first part of Proposition 6 occurs when $\bar{c}$ is such that $1-\varpi_{E}(\bar{c}) E\left(\mathrm{D}_{1}\right)=0$, in which case the ESF point is $E^{-1}\left(\mathrm{D}_{1}\right)\left(1-A_{a}^{+}\right)$, whose risky part has a zero average cost. Nevertheless, we can establish a link between this element of the ESF and the asymptotes of the URF, as it was the case of the CRF. Similarly, the exception to the second part of Proposition 6 occurs when $\bar{\nu}$ is such that $E\left(\mathrm{~F}_{1}\right)-\omega_{U}(\bar{v}) E\left(\mathrm{D}_{1}\right)=0$, in which case the URF point is $E^{-1}\left(\mathrm{D}_{1}\right) E\left(\mathrm{~F}_{1}\right) p_{e}^{+}$, which does not have any weight on $p_{e}^{*}$. But again, we can establish a link between this element of the URF and the asymptotes of the ESF, as it was the case of the CSF.

In any case, while the URF characterises the optimal unconditional risk-return trade offs, its dual, i.e. the ESF, only provides suboptimal constraints on asset pricing models.

\section{Passive Mean-Variance Frontiers}

Let us now study those situations in which investors do not use the information available at the trading date $\mathbf{l}$ in constructing their portfolio weights, so that they rely on passive strategies. To be internally consistent, we will work with unconditional moments in such a framework, which corresponds to the last three columns in Table 1. At first sight, it may seem irrelevant to study passive strategies in the presence of conditioning information. However, most of the empirical 
work on mean-variance frontiers relies on passive strategies of managed portfolios as a way of approximating the complexity of active strategies without running the risk of misspecifying the conditional distribution of asset returns (see chapter 8 in Cochrane (2001) for a justification).

\subsection{Passive Payoff Space and SDFs}

Given a vector of asset payoffs $\mathbf{x}$, we define the passive payoff space $P \subseteq P_{a}$ as the space ${ }^{14}$ of constant weight portfolios

$$
P=\left\{p \in P_{a}: p=\mathbf{w}^{\prime} \mathbf{x}, \mathbf{w} \in \mathbb{R}^{N}\right\} .
$$

In our multinomial illustration described in section $2.2, P$ represents strategies where $\mathbf{w}_{1}^{1}=$ $\mathbf{w}_{1}^{2}=\mathbf{w}_{1}^{3}=\mathbf{w}_{\mathbf{1}}^{4}=\mathbf{w}$.

In this context, we can define the passive representing portfolios as the two unique elements $\left(p^{+}, p^{*}\right)$ of $P$ that represent average mean and cost over $P$. That is,

$$
E(p)=E\left(p^{+} p\right), \quad C(p)=E\left(p^{*} p\right), \quad \forall p \in P,
$$

whence it is easy to see that

$$
p^{+}=\mathbf{x}^{\prime} \boldsymbol{\Gamma}^{-1} \boldsymbol{\nu}, \quad p^{*}=\mathbf{x}^{\prime} \boldsymbol{\Gamma}^{-1} \mathbf{c} .
$$

Note that $p^{+}$is the unconditional projection of the safe payoff $x_{0}=1$ onto $P$. As for $p^{*}$, we can interpret it as the unconditional projection of a new type of SDFs that we will call passive SDFs onto $P$. More formally, we define passive SDFs as those random variables $m \in I_{2}$ that price any payoff in $P$ correctly on average, i.e.:

$$
E(m p)=C(p), \quad \forall p \in P .
$$

Recall that active SDFs satisfy an analogous equation for a richer set of payoffs, namely the whole $P_{a}$. Passive SDFs are equivalently characterised by the condition

$$
E(m \mathbf{x})=\mathbf{c}
$$

because they satisfy $E\left[\mathbf{w}^{\prime}(m \mathbf{x}-\mathbf{c})\right]=0$ for any $\mathbf{w} \in \mathbb{R}^{N}$. In our multinomial illustration described in section 2.2, passive SDFs satisfy

$$
E(m \mathbf{x})=\sum_{k=1}^{4} \pi^{k} \mathbf{c}_{\mathbf{1}}^{k} .
$$

\footnotetext{
${ }^{14}$ Like $P_{e}, P$ also has the structure of a subspace of $P_{a}$ with respecto to unconditional linear combinations.
} 


\subsection{Passive Return and SDF Frontiers: PRF and PSF}

Hansen and Jagannathan (1991) define a frontier, which we will label as the Passive Return Mean-Variance Frontier (PRF), made up of those passive portfolio strategies that solve a problem analogous to (19) but this time defined over $P$ :

$$
\min _{p \in P} E\left(p^{2}\right) \quad \text { s.t. } \quad E(p)=\bar{\nu}, \quad C(p)=1 .
$$

Importantly, the elements of the PRF will generally be extended returns instead of returns since the cost constraint in (31) is stated as an average. In this sense, note that it is not possible to construct proper returns with passive strategies unless the original payoffs $\mathbf{x}$ have nonrandom prices, a special case that will be analysed in detail in sections 6.4 and 6.5.2.

The results in Hansen and Jagannathan (1991) imply that the PRF can be represented by the (unconditionally) orthogonal decomposition

$$
\begin{aligned}
p_{P}(\bar{\nu}) & =R^{*}+\omega(\bar{\nu}) A^{+}, \\
\omega(\bar{\nu}) & =\frac{\bar{\nu}-\mathrm{A} / \mathrm{C}}{\mathrm{B}-\mathrm{A}^{2} / \mathrm{C}},
\end{aligned}
$$

where

$$
R^{*}=\frac{1}{\mathrm{C}} p^{*}
$$

is the extended return associated to $p^{*}$,

$$
A^{+}=p^{+}-\frac{\mathrm{A}}{\mathrm{C}} p^{*}
$$

is the extended arbitrage portfolio given by the residual of the unconditional projection of $p^{+}$ onto $\left\langle p^{*}\right\rangle$, and

$$
\begin{gathered}
\mathrm{A}=\boldsymbol{\nu}^{\prime} \boldsymbol{\Gamma}^{-1} \mathbf{c}=E\left(p^{*}\right)=C\left(p^{+}\right)=E\left(p^{+} p^{*}\right), \\
\mathrm{B}=\boldsymbol{\nu}^{\prime} \boldsymbol{\Gamma}^{-1} \boldsymbol{\nu}=E\left(p^{+}\right)=E\left(p^{+2}\right), \\
\mathrm{C}=\mathbf{c}^{\prime} \boldsymbol{\Gamma}^{-1} \mathbf{c}=C\left(p^{*}\right)=E\left(p^{* 2}\right) .
\end{gathered}
$$

As expected, the PRF also shows unconditional two fund spanning. In addition, given that the second moment of portfolios on the PRF is

$$
E\left[p_{P}^{2}(\bar{\nu})\right]=\frac{1}{\mathrm{C}}+\omega^{2}(\bar{\nu})\left(\mathrm{B}-\frac{\mathrm{A}^{2}}{\mathrm{C}}\right),
$$

the PRF will be a hyperbola in $[\sqrt{\operatorname{Var}(p)}, E(p)]$ space. In this sense, we can easily prove that the PRF will be to the right of the ERF on this space because (35) and (19) are identical 
programs, except that the former is defined on the narrower feasible set of passive strategies, $P \subseteq P_{a}$. In contrast, the PRF and URF may in principle cross. As a result, the PRF is not necessarily a relevant object for an investor when it is not constructed from constant cost payoffs, neither can it be used to place a bound on the URF in that case.

On the other hand, Hansen and Jagannathan (1991) define a frontier that we will label as the Passive SDF Mean-Variance Frontier (PSF), which puts the highest variance bound on those univariate random variables that price on average any portfolio with constant weights. These random variables, though, are generally passive SDFs, and not necessarily valid SDFs, since they may not price correctly portfolios with time-varying weights. More formally, the PSF is given by the set of scalar random variables that solve the problem

$$
\min _{m \in I_{2}} E\left(m^{2}\right) \quad \text { s.t. } \quad E(m)=\bar{c}, \quad E(m \mathbf{x})=\mathbf{c} .
$$

Their results directly imply that the PSF can be represented as

$$
\begin{aligned}
m_{P}(\bar{c}) & =p^{*}+\varpi(\bar{c}) E^{+}, \\
\varpi(\bar{c}) & =\frac{\bar{c}-\mathrm{A}}{1-\mathrm{B}},
\end{aligned}
$$

where $p^{*}$ is defined in (30) and

$$
E^{+}=1-p^{+}
$$

is the residual of the unconditional projection of 1 onto $P$. Finally, Hansen and Jagannathan also show that there is unconditional two fund spanning in this frontier too.

Given that the second moment of SDFs on the PSF are

$$
E\left[m_{P}^{2}(\bar{c})\right]=\mathrm{C}+\varpi^{2}(\bar{c})(1-\mathrm{B}),
$$

the PSF will also be a hyperbola in $[E(m), \sqrt{\operatorname{Var}(m)}]$ space. As Gallant, Hansen and Tauchen (1990) point out, the PSF will be below the USF in this space because (35) and (16) are identical programs, except for the fact that the latter adds more demanding pricing constraints. While the USF prices correctly on average every payoff in $P_{a}$, the PSF only prices correctly on average payoffs in $P \subseteq P_{a}$. Hence, the PSF places a lower bound on the USF. In contrast, the ESF and PSF may in principle cross since the elements of each frontier yield average prices in a subspace of payoffs that does not necessarily contain the other one. Consequently, the ESF does not necessarily sharpen the SDF bounds provided by the PSF. 


\subsection{Duality Between PRF and PSF}

One of the most cited results in Hansen and Jagannathan (1991) is that there is an elementby-element duality between the PRF and PSF. Specifically, these authors show that the traded component of any element of the $(35) m_{P}(\bar{c})$ such that $\mathrm{C}-\varpi(\bar{c}) \mathrm{A} \neq 0$ will be unconditionally proportional to some element of the $(31) p_{P}(\bar{v})$ if and only if $\bar{v}$ and $\bar{c}$ satisfy

$$
\varpi(\bar{c})-\mathrm{A} \varpi(\bar{c}) \omega(\bar{v})+\mathrm{C} \omega(\bar{v})=0 .
$$

Likewise, any element of the PSF $p_{P}(\bar{v})$ such that $1-\omega(\bar{v}) \mathrm{A} \neq 0$ will be unconditionally proportional to the traded part of some element of the $m_{P}(\bar{c})$ if and only if $\bar{v}$ and $\bar{c}$ also satisfy (38).

The two exceptions to this duality are easy to understand by a direct translation of the comments made for conditional frontiers after Proposition 1. Similarly, the geometry of duality and its exceptions are analogous to Figure 2.

\subsection{Passive Frontiers with Returns}

Let us focus on the last column in Table 1, when $\mathbf{x}$ is effectively an $N \times 1$ vector of gross returns $\mathbf{R}$, possibly after scaling by their non-random cost.

As is well known, the PRF coincides with the Markowitz (1952) frontier in this case. Further, the PRF will be a constrained version not only of the ERF but also of the URF because any extended return in $P$ will also be a return in those circumstances. Therefore, we will come across the ERF, the URF and the PRF as we go from left to right on the $[\sqrt{\operatorname{Var}(p)}, E(p)]$ space, as in Figure 4. In those circumstances, we can understand the PRF as providing a weak lower bound on the actual risk-return trade-offs that investors face, which are described by the URF, not the ERF.

Let us turn to SDF frontiers. If $\mathbf{c}_{1}$ is nonrandom then the ESF will be a constrained version of the PSF because $P \subset P_{e}$ in this special case. Therefore, as we move downwards on the $[\sqrt{\operatorname{Var}(p)}, E(p)]$ space we will come across the USF, the ESF and finally the PSF, as illustrated in Figure 4. 

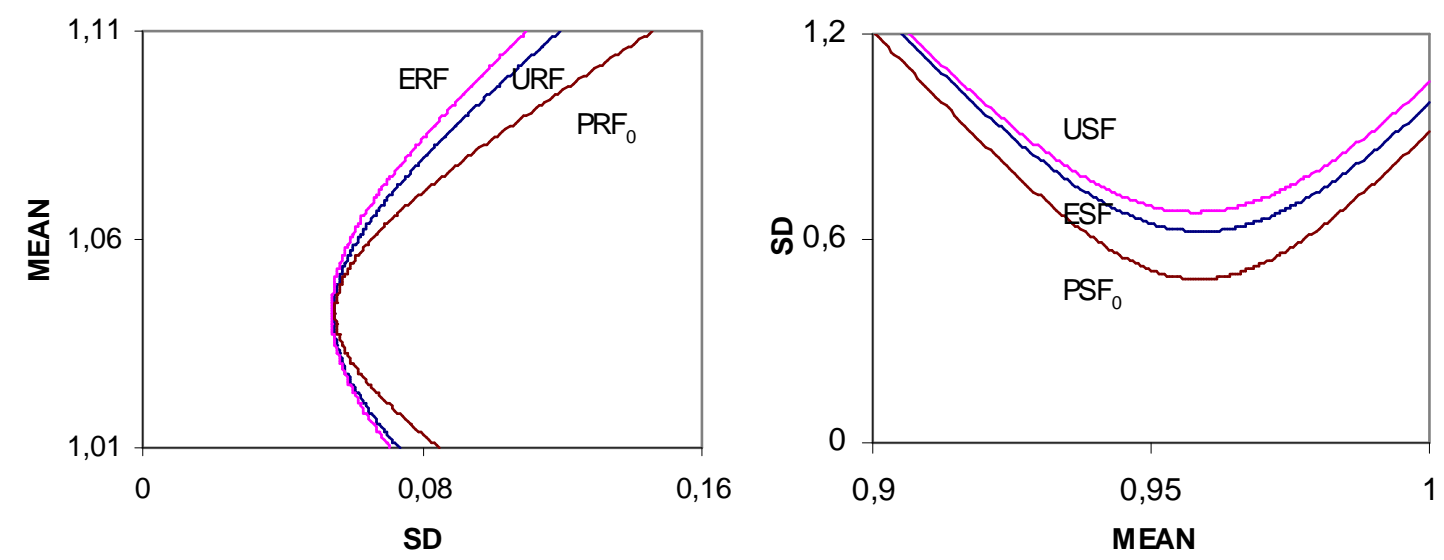

Figure 4: Passive frontiers based on returns alone $\left(\mathrm{PRF}_{0}\right.$ and $\left.\mathrm{PSF}_{0}\right)$ jointly with unconditional and extended frontiers. Portfolio frontiers on the left and SDF frontiers on the right.

\subsection{Passive Frontiers with Managed Portfolios}

\subsubsection{Unrestricted Managed Portfolios}

In practice, the passive payoff space $P \subset P_{a}$ spanned from the return vector $\mathbf{R}$ only may be too narrow relative to $P_{a}$, which is the relevant space of active strategies available to investors. For that reason, Hansen and Jagannathan (1991) also relied on an alternative empirical approach that augments the payoff space $P$ with the payoffs of some managed portfolios in an attempt to approximate $P_{a}$ as closely as possible, and thereby sharpen both the PRF and PSF. This strategy, which has gained much popularity in empirical work because it avoids the (mis)specification of a conditional model for asset payoffs, corresponds to the penultimate column of Table 1.

As an extreme example, consider the enlarged payoff vector

$$
\mathbf{x}=\left(\begin{array}{llll}
\xi^{1} \mathbf{R}^{\prime} & \xi^{2} \mathbf{R}^{\prime} & \xi^{3} \mathbf{R}^{\prime} & \xi^{4} \mathbf{R}^{\prime}
\end{array}\right)^{\prime}
$$

in the multinomial example of section 2.2. In this case, it is easy to see that $P=P_{a}$, in which case $P_{e} \subset P$. The scaled payoffs $\xi^{k} \mathbf{R}$, which are called managed portfolios in the empirical literature since their scale belongs to $I_{1}$, are no longer returns since their true cost

$$
C\left(\mathbf{x} \mid G_{1}\right)=\left(\begin{array}{llll}
\xi^{1} \ell^{\prime} & \xi^{2} \ell^{\prime} & \xi^{3} \ell^{\prime} & \xi^{4} \ell^{\prime}
\end{array}\right)^{\prime}
$$

varies with the values of the signals, where $\ell$ is an $N \times 1$ vector of ones. As far as the unconditional and extended frontiers discussed in the previous versions is concerned, though, the use of $\mathbf{x}$ or $\mathbf{R}$ leads to the same answer because $\langle\mathbf{x}\rangle_{1}=\langle\mathbf{R}\rangle_{1}$, i.e. $\mathbf{x}$ does not increase the payoff spaces $P_{e}$ 
and $P_{a}$. Given that $P=P_{a}$ in this extreme example, a sharpened PRF (31) constructed with this $\mathbf{x}$ instead of the initial $\mathbf{R}$ is exactly equal to the ERF in (19), while a sharpened PSF in (35) constructed with this $\mathbf{x}$ is exactly equal to the USF in (16). In other words, the fifth column in Table 1 coincides with the third column in this case.

Therefore, the use of unrestricted managed portfolios and passive frontiers gives a relevant object when applied to SDF frontiers, but not when applied to portfolio frontiers. As we have repeatedly mentioned, PRFs constructed from unrestricted managed portfolios are useless from the vantage point of an investor because they are made up of portfolios whose average cost is 1 , but whose real cost depends on the value of the signals.

The difference between the PRF and the Markowitz frontier in the case of managed portfolios does not seem to be widely known, perhaps because empirical work initially relied on returns. However, more recent empirical work on the PSF tends to rely on managed portfolios, which are payoffs with possibly non-constant cost.

Of course, in actual empirical work it is not usually possible to explore all the relevant managed portfolios. Consequently, the sharpened PRF and PSF will rely on passive strategies based on a subset of the vector of all conceivable managed portfolios. For instance, we could use

$$
\mathbf{x}=\left(\begin{array}{ll}
\mathbf{R}^{\prime} & \xi^{1} \mathbf{R}^{\prime}
\end{array}\right)^{\prime}
$$

which (passively) spans a payoff space $P$ that is richer than the one based on $\mathbf{R}$, but poorer than $P_{a}$. Specifically, in this example $P$ is constrained to those active strategies in which $\mathbf{w}_{\mathbf{1}}^{2}=\mathbf{w}_{\mathbf{1}}^{3}=\mathbf{w}_{\mathbf{1}}^{4}$. In $[\sqrt{\operatorname{Var}(p)}, E(p)]$ space, a sharpened PRF constructed with such an $\mathbf{x}$ will lie between the ERF and the PRF based on $\mathbf{R}$ alone, and could cross the URF. On the other hand, a sharpened PSF constructed with such an $\mathbf{x}$ will be between the USF and the PSF based on $\mathbf{R}$ in $[E(m), \sqrt{\operatorname{Var}(m)}]$ space, and might even cross the ESF.

Figure 5 shows two alternative ways of partially sharpening the PRF and the PSF by

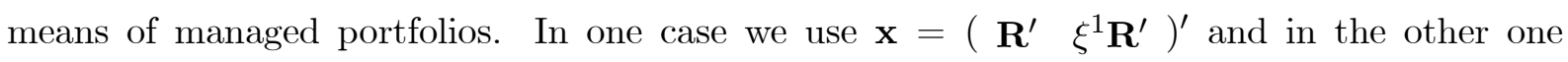
$\mathbf{x}=\left(\begin{array}{ll}\mathbf{R}^{\prime} & \xi^{4} \mathbf{R}^{\prime}\end{array}\right)^{\prime}$. The PRF that relies on $\xi^{1}$ is between the PRF based on returns alone and the URF, but the PRF that relies on $\xi^{4}$ is very close to the ERF, crossing the URF around its global minimum variance point. More importantly, the PSF that relies on $\xi^{1}$ is between the PSF that relies on returns alone and the ESF, but the PSF that relies on $\xi^{4}$ is very close to the USF, crossing the ESF out of the relevant range of points in this plot. This figure confirms that the ESF does not necessarily improve upon a simple application of managed portfolios. 

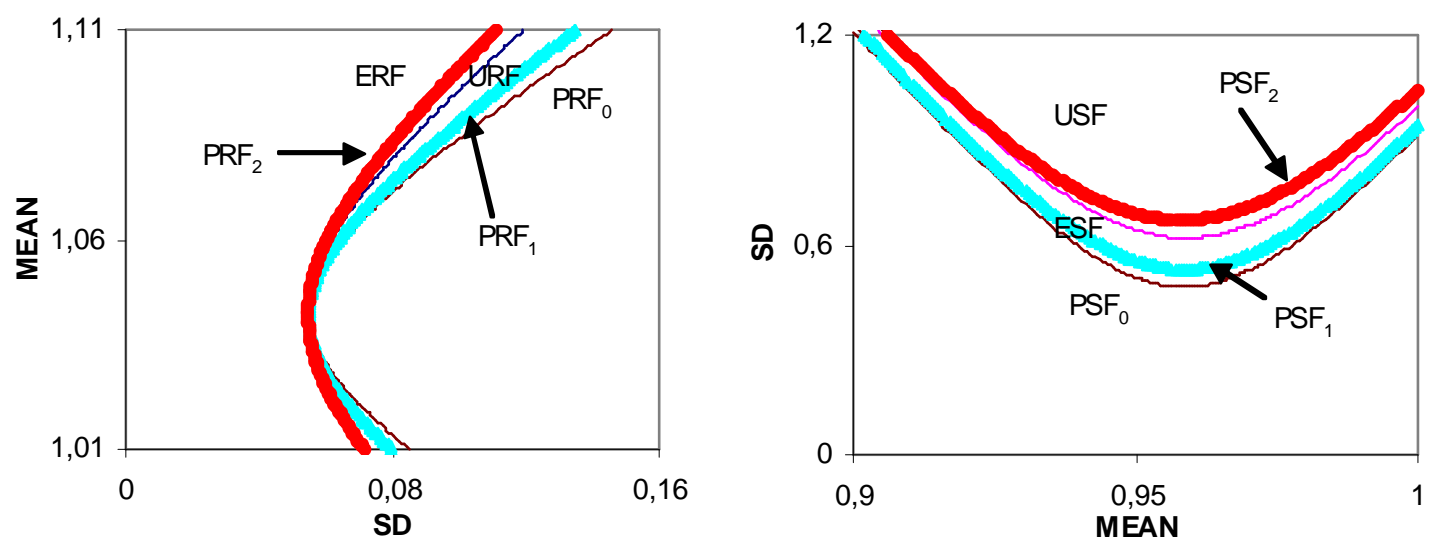

Figure 5: Passive frontiers with unrestricted managed portfolios. $\mathrm{PRF}_{0}$ and $\mathrm{PSF}_{0}$ are based on returns alone, $\mathrm{PRF}_{1}$ and $\mathrm{PSF}_{1}$ are based on returns and $\xi^{1}$, and $\mathrm{PRF}_{2}$ and $\mathrm{PSF}_{2}$ are based on returns and $\xi^{4}$. Portfolio frontiers on the left and SDF frontiers on the right.

\subsubsection{Managed Portfolios of Constant Cost}

Imagine now that we restrict the managed portfolios that we use to have constant cost, which corresponds to the fourth column in Table 1. If the primitive assets in (24) are a vector of returns $\mathbf{R}$, then we can represent any $p \in P_{e}$ as

$$
p=R_{1} w+\mathbf{e}_{-1}^{\prime} \mathbf{w}_{\mathbf{1},-1}, \quad w \in \mathbb{R}, \quad \mathbf{w}_{\mathbf{1},-1} \in I_{1},
$$

where $\mathbf{e}_{-1}=\mathbf{R}_{-1}-R_{1} \ell_{-1}$. This expression motivates the approximation of $P_{e}$ by means of passive strategies on an augmented set of payoffs used by Bansal, Dahlquist, and Harvey (2004) and Brandt and Santa-Clara (2006) among others, who re-scale $\mathbf{e}_{-1}$ with random variables that belong to $I_{1}$. Note that such a space would be the relevant one for a passive investor who has access to active funds.

As an extreme example, consider the enlarged payoff vector

$$
\mathbf{x}=\left(\begin{array}{lllll}
R_{1} & \xi^{1} \mathbf{e}_{-1}^{\prime} & \xi^{2} \mathbf{e}_{-1}^{\prime} & \xi^{3} \mathbf{e}_{-1}^{\prime} & \xi^{4} \mathbf{e}_{-1}^{\prime}
\end{array}\right)^{\prime},
$$

in the multinomial example of section 2.2. In this case, it is easy to see that $P=P_{e} \subset P_{a}$, which means that a sharpened PRF (31) constructed with this $\mathbf{x}$ instead of the initial $\mathbf{R}$ is exactly equal to the URF in (14), while a sharpened PSF in (35) constructed with this $\mathbf{x}$ is exactly equal to the ESF in (25). In other words, the fourth column in Table 1 coincides with the second column in this case. 
Obviously, passive frontiers that rely on a subvector of (39) will lie between the PRF based on $\mathbf{R}$ alone and the URF, and between the PSF based on $\mathbf{R}$ alone and the ESF. Therefore, the use of constant cost managed portfolios and passive frontiers gives a relevant object when applied to return frontiers, but not when applied to SDF frontiers.

\subsection{3 "Optimal" Managed Portfolios}

Given that the choice of managed portfolios is empirically relevant, Ferson and Siegel (2003) and Bekaert and Liu (2004) sharpen the PSF bounds obtained from $\mathbf{R}$ with some carefully chosen managed portfolios, as indicated at the bottom of Table 1.

Ferson and Siegel (2003) construct a PSF from any two arbitrary returns on the URF (15). More formally, if we call $\bar{\nu}_{1}$ and $\bar{\nu}_{2}$ the two chosen expected returns, with $\bar{\nu}_{1} \neq \bar{\nu}_{2}$, then we can express their problem as (35) with

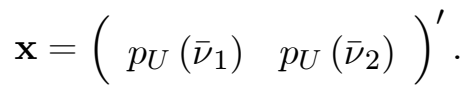

Ferson and Siegel (2003) motivate this choice of payoffs on the grounds that these two portfolios optimally use conditioning information from the point of view of an unconditional mean-variance investor. However, Proposition 6 implies that their procedure generates the ESF $(25),{ }^{15}$ so that they effectively bound the unconditional variances of extended SDFs, which are not necessarily true SDFs because they will not generally price correctly on average managed portfolios with random cost. Moreover, a simple application of (unrestricted) managed portfolios may even improve upon the ESF, as Figure 5 illustrates.

Bekaert and Liu (2004) consider a different type of optimality in choosing their managed portfolios. In particular, they first use (35) to obtain the minimum unconditional variance of any SDF $m$ with unconditional mean $\bar{c}$ that prices correctly on average some single payoff $x$. Given that such a bound depends not only on $\bar{c}$ but also on $x$, Bekaert and Liu (2004) then find the managed portfolio $x(\bar{c}) \in P_{a}$ that yields the highest possible bound. In this way, they generate the whole USF by means of a PSF-like object that prices on average a "single" payoff that nevertheless changes with $\bar{c}$. Strictly speaking, therefore, the frontier that they obtain is not a standard PSF (35).

Using our notation, we can express the optimal payoff $x(\bar{c})$ of Bekaert and Liu (2004) as the traded component of a particular point on the USF (17), and the problem that they solve as

\footnotetext{
${ }^{15}$ Similarly, a simple application of unconditional two-fund spanning shows that a PRF problem (31) with this $\mathbf{x}$ is equivalent to the URF (14).
} 
a transformation of problem (35) where the single managed portfolio that they consider for a fixed $\bar{c}$ will be

$$
x(\bar{c})=p_{a}^{*}-\varpi_{U}(\bar{c}) p_{a}^{+}=m_{U}(\bar{c})-\varpi_{U}(\bar{c}) .
$$

This expression of $x(\bar{c})$ motivates an interpretation of the equality between their bounds and the USF (16) by means of a dual's dual argument since $x(\bar{c})$ is unconditionally proportional to an element on the ERF. ${ }^{16}$ In any case, Proposition 4 shows that one should be careful when trying to use the frontier obtained by Bekaert and Liu (2004) to guide dynamic asset allocation because its dual object is the ERF, which is not the relevant object for investment decisions since its elements are not necessarily returns.

Finally, it is important to note that Ferson and Siegel (2003) and Bekaert and Liu (2004) obtained different SDF bounds because they applied their methods to different payoffs, not because their methods were fundamentally different. In particular, if $\mathbf{x}$ contained two extended returns on the ERF (20) instead of the two returns on the URF in (40), then the solution to the Ferson and Siegel's approach would be the USF. ${ }^{17}$ Similarly, if instead of (41) we used $x(\bar{c})=m_{E}(\bar{c})-\varpi_{E}(\bar{c})$, which is the traded component of a point on the ESF (26) with mean $\bar{c}$, then the solution of Bekaert and Liu's approach would be the ESF, as Abhyankar, Basu and Stremme (2007) show. $^{18}$

\section{The Riskless Asset Case}

In the remaining of the paper, we will focus on three special cases in which it becomes easier to characterise the different frontiers discussed in the previous sections. We will initially study the potentially relevant situation in which a safe asset exists, and leave the cases of equal expected returns and arbitrage portfolios for the next section.

Imagine that investors have access to a set of assets $\mathbf{y}$ that includes not only the original risky asset payoffs in $\mathbf{x}$, but also the safe payoff $x_{0}=1$, so that $\mathbf{y}^{\prime}=\left(x_{0}, \mathbf{x}^{\prime}\right)$. In this context, $Q_{a}=\langle\mathbf{y}\rangle_{1}$ will be an enlarged payoff space such that $Q_{a} \supset P_{a}$. In addition, we define the corresponding

\footnotetext{
${ }^{16}$ In particular, the boundary point associated to $\bar{c}$ obtained by Bekaert and Liu (2004) must necessarily belong to the PSF based on the trivial passive portfolio space that simply scales $x(\bar{c})$ with a real number. Therefore, it must have a dual point on the corresponding PRF, which will trivially coincide with the element given by the extended return of $x(\bar{c})$. As shown in the proof of Proposition 4, the extended return of $x(\bar{c})$ (which is the traded part of $\left.m_{U}(\bar{c})\right)$ can be expressed as $R_{e}^{*}+\omega_{E}(\bar{v}) A_{e}^{+}$, which is a point on the ERF $(20)$. But since we know that any point on the ERF has a dual point on the USF (17), the Bekaert and Liu's boundary point from which we started must be a point on the USF.

${ }^{17}$ For analogous reasons, the PRF based on $P=\left\langle p_{a}^{+}, p_{a}^{*}\right\rangle$ will be equal to the ERF.

${ }^{18}$ These authors compare the theoretical and empirical properties of Ferson and Siegel's and Bekaert and Liu's SDF bounds by focusing on the corresponding $x(\bar{c})^{\prime} s$.
} 
price vector as $\mathbf{d}_{1}^{\prime}=\left(c_{01}, \mathbf{c}_{1}^{\prime}\right)$, while the vector of average prices will be $\mathbf{d}=E\left(\mathbf{d}_{\mathbf{1}}\right)$. The first entry of $\mathbf{d}$ is the pseudo-price of the safe payoff $c_{0}=E\left(c_{0_{1}}\right)$. On this basis, we can define the conditionally safe return and the extended return associated to the riskless asset as

$$
R_{0}=\frac{1}{c_{01}} \in I_{1}, \quad S_{0}=\frac{1}{c_{0}} \in \mathbb{R}
$$

respectively. We say that the safe asset is unconditionally riskless when $c_{01}=c_{0}$, so that $R_{0}=S_{0}$. For simplicity, in constructing Figures 6-9 we maintain the assumption that there is an unconditionally safe asset.

The conditional mean and cost active representing portfolios in the payoff space $Q_{a}$ will be

$$
q_{a}^{+}=1, \quad q_{a}^{*}=p_{a}^{*}+\left[\frac{c_{01}-\mathrm{A}_{1}}{1-\mathrm{B}_{1}}\right] E_{a}^{+}
$$

respectively. Note that $q_{a}^{+}$is trivially the conditional projection of $x_{0}$ onto $Q_{a}$, and hence the corresponding residual will be 0 . On the other hand, $q_{a}^{*}$ is the conditional projection of any valid SDF onto $Q_{a}$, which obviously coincides with $m_{C}\left(c_{01}\right)$ (see (11)).

In the rest of this section we will describe in detail the different mean-variance frontiers that one can construct, with a special emphasis on their shape, the relationship between frontiers with and without a safe asset, and a geometrical interpretation of duality by means of Sharpe ratios.

\subsection{Conditional Frontiers}

The mean-variance problems that we must solve in this context are very similar to the analogous problems without a safe payoff discussed in section 3. In particular, the elements of the CRF will solve the same problem as (6), except that $p$ is allowed to belong to the enlarged conditional span $Q_{a}$. Therefore, it is not surprising that (7) is still valid after the introduction of a safe payoff if we simply replace $p_{a}^{+}$and $p_{a}^{*}$ in (4) with $q_{a}^{+}$and $q_{a}^{*}$ in (43), respectively. Obviously, the notation $\left(\mathrm{A}_{1}, \mathrm{~B}_{1}, \mathrm{C}_{1}\right)$ and its variants should also be adapted to $q_{a}^{+}$and $q_{a}^{*}$.

As expected, the elements of the CRF lie along two straight lines in $\left[\sqrt{\operatorname{Var}\left(p \mid G_{1}\right)}, E\left(p \mid G_{1}\right)\right]$ space for each possible signal value in $G_{1}$. Moreover, those two lines intersect on the vertical axis at $R_{0}$, which was defined in (42). The first panel of Figure 6 illustrates the CRF for each signal value of the multinomial illustration described in section 2.2.

In addition, there is a conditional mean profile $\bar{\nu}_{1}$ such that the weight of the CRF on the conditionally safe payoff $x_{0}$ will be identically 0 for every possible signal realisation, which 
implies that it will be equal to the CRF without a safe asset $p_{C}\left(\bar{\nu}_{1}\right)$ at that point. This shared element is usually referred to as the tangency portfolio. Figure 7 shows the connection between the CRFs with and without the safe asset at a particular signal value.
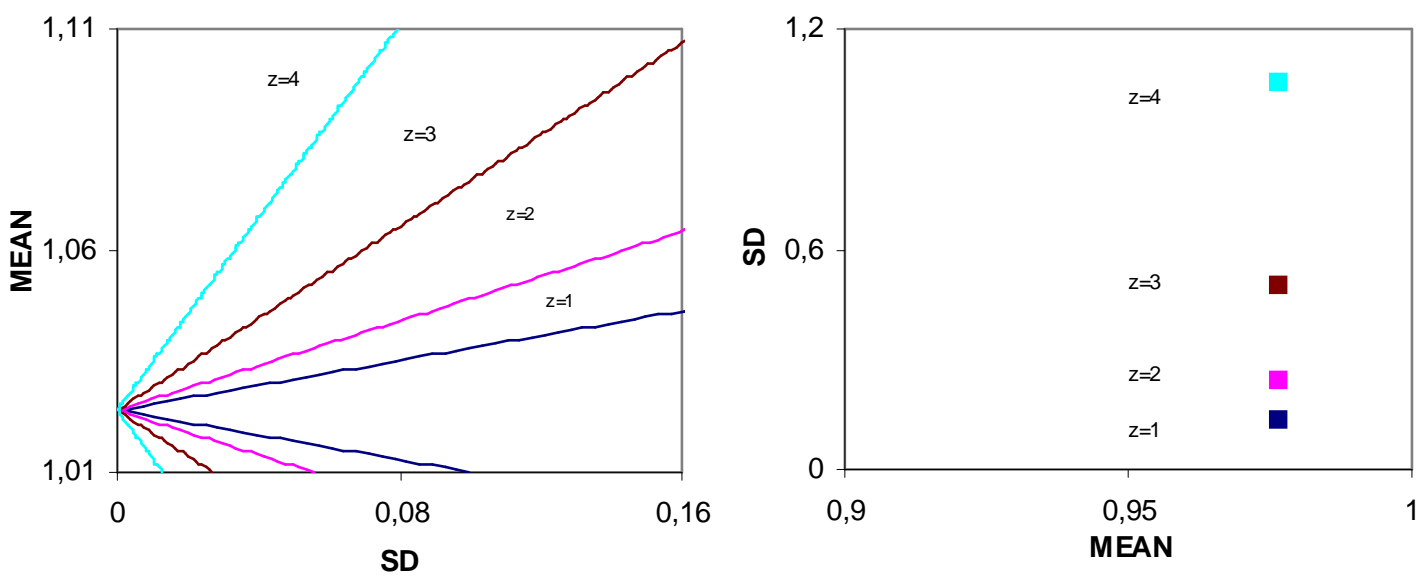

Figure 6: Mean-variance frontiers conditional on specific signal values in the presence of an unconditionally riksless asset. Portfolio frontiers on the left and SDF frontiers on the right.
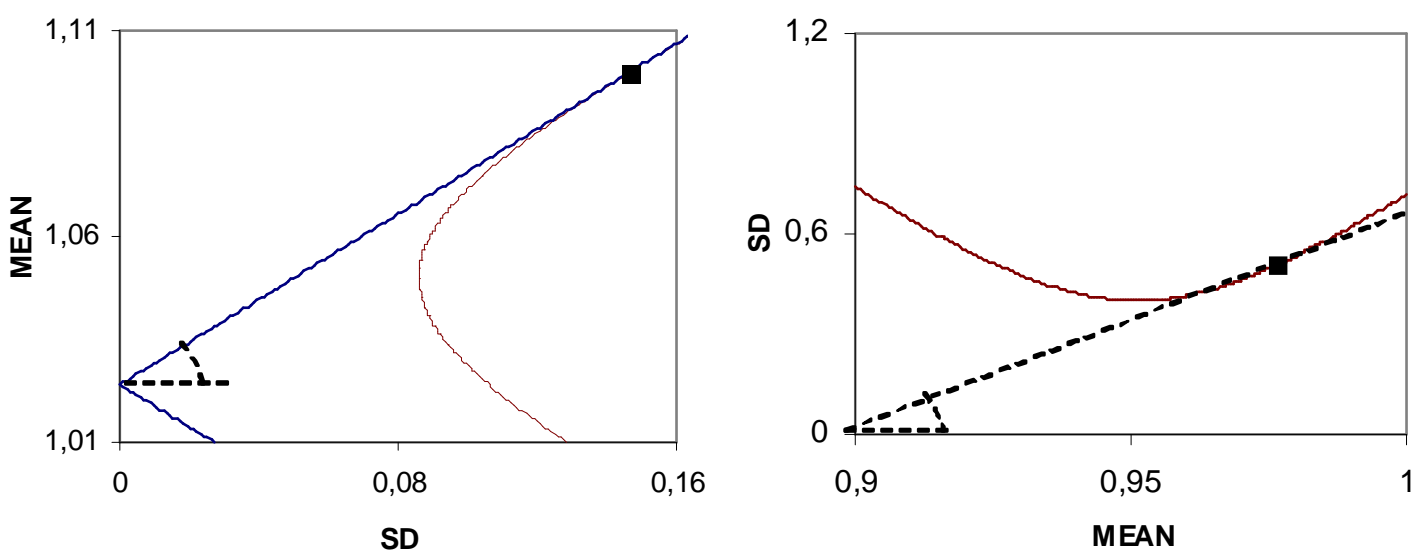

Figure 7: Duality between conditional frontiers at a particular signal value in the presence of an unconditionally riskless asset. Portfolio frontiers on the left and SDF frontiers on the right.

Similarly, the elements of the CSF solve the same problem as in (10), although this time they will also have to satisfy the additional pricing restriction $E\left(m x_{0} \mid G_{1}\right)=c_{01}$. But since the only conditional mean profile $\bar{c}_{1}$ for which the mean and pricing constraints will be compatible is $c_{01}$, 
which is the conditional cost profile of the safe payoff, the CSF will be given by the singleton $q_{a}^{*}$. Not surprisingly, $q_{a}^{*}$ also belongs to the CSF without a safe payoff since it coincides with $m_{C}\left(c_{01}\right)$. The second panel of Figure 6 illustrates the CSF for each signal value, while Figure 7 shows the connection between the CSFs with and without the safe asset for a particular signal value.

In this context, the duality between the CRF and CSF is trivial since the latter is fully traded and its corresponding return is

$$
S_{a}^{*}=\frac{1}{E\left(q_{a}^{* 2} \mid G_{1}\right)} q_{a}^{*}
$$

Alternatively, we can illustrate the duality between the CRF and CSF by adapting the geometrical argument on mean-standard deviation spaces that relates the PRF and PSF in Hansen and Jagannathan (1991). Specifically, if we take into account that $S_{a}^{*}$ lies on the inefficient branch of the CRF for each signal's realisation, then the optimal conditional Sharpe ratio on the $\mathrm{CRF}$ will be equal to the slope of the ray that joins the origin with the single point on the CSF, so that

$$
S R_{1}=\frac{\left|E\left(S_{a}^{*} \mid G_{1}\right)-R_{0}\right|}{\operatorname{Var}^{1 / 2}\left(S_{a}^{*} \mid G_{1}\right)}=\frac{\operatorname{Var}^{1 / 2}\left(q_{a}^{*} \mid G_{1}\right)}{E\left(q_{a}^{*} \mid G_{1}\right)},
$$

as illustrated in Figure 7.

\subsection{Unconditional Frontiers}

When a safe payoff is available, the elements of the URF solve the same problem as (14), except that $p$ is allowed to belong to the enlarged conditional span $Q_{a}$. Again, (15) is still valid after the introduction of a safe payoff if we simply replace $p_{a}^{+}$and $p_{a}^{*}$ in (4) with $q_{a}^{+}$and $q_{a}^{*}$ in (43), respectively. Figure 8 shows the URFs with and without a safe asset.

In this context, we find two facts that contradict conventional wisdom on mean-variance frontiers with a safe asset. First, the elements of the URF do not lie along two straight lines in $[\sqrt{\operatorname{Var}(p)}, E(p)]$ space if $R_{0}$ is random, which means that there is not a unique optimal risk-return trade-off, nor is $R_{0}$ a frontier portfolio in that case (see Hansen and Richard (1987)). But if the conditionally safe return is also unconditionally riskless because the price of $x_{0}$ is constant, then the URF will indeed consist of two straight lines that intersect on the vertical axis at $R_{0}=S_{0}$, where both $R_{0}$ and $S_{0}$ were defined in (42).

Second, there is no tangency portfolio irrespective of whether $R_{0}=S_{0}$, because the risky component of the elements of the augmented URF will not be conditionally proportional to the 
$p_{U}(\bar{\nu})^{\prime} s$ in (15) that conform the original URF. Intuitively, the reason is that investors can trade random amounts of the safe asset by means of active strategies, which implies that even a nonrandom $R_{0}$ leads to additional investment opportunities in terms of unconditional moments. As a result, the Sharpe ratios that Bekaert and Liu (2003) and Abhyankar, Basu and Stremme (2007) consider must be interpreted with some care, as they relate to passive strategies that combine an unconditionally riskless asset (traded or fictitious) with a portfolio on the URF of risky assets alone. As a result, those Sharpe ratios underestimate the maximum unconditional risk-return trade-off that can be achieved through active portfolio strategies.
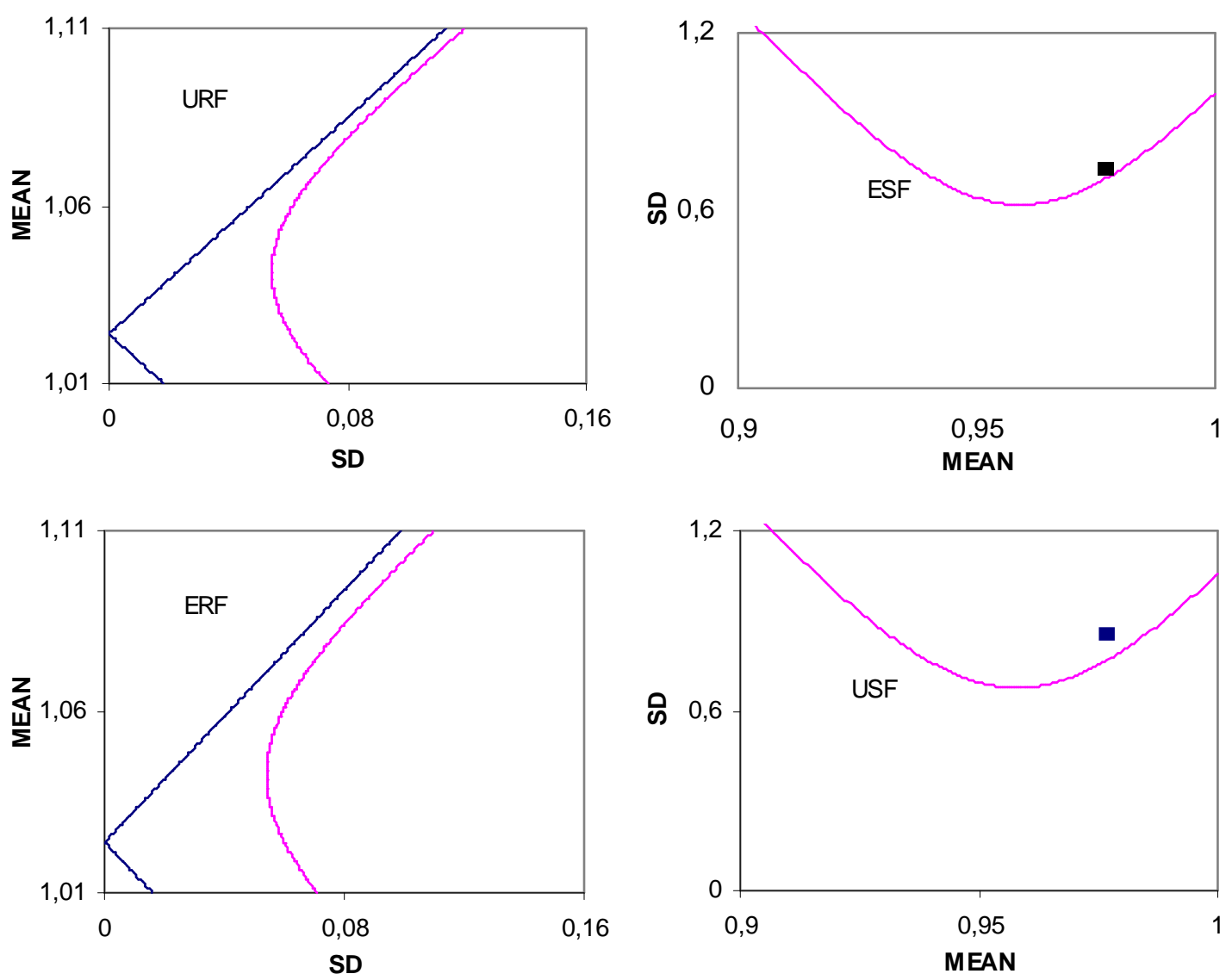

Figure 8: Unconditional and extended frontiers with and without an unconditionally riskless asset. Portfolio frontiers on the left and SDF frontiers on the right.

As for the elements of the USF, they solve the same problem as in (16), but with the additional pricing restriction $E\left(m x_{0} \mid G_{1}\right)=c_{01}$. Once again, the only $\bar{c}$ for which the mean and pricing constraints will be compatible is $c_{0}$, which is the pseudo-price of the safe payoff. As a 
result, the USF will also be a singleton. In fact, the only difference between the problems that define the USF and the CSF is the criterion function, as the former minimises $E\left(m^{2}\right)$ while the latter minimises $E\left(m^{2} \mid G_{1}\right)$. Therefore, a simple application of the law of iterated expectations implies that the solution to both problems must be the same since the corresponding feasible sets are equal and $m^{2} \geq 0$. In other words, the USF will also consist of $q_{a}^{*}$ only. However, this portfolio does not generally coincide with any $m_{U}(\bar{c})$ (see $(17)$ ) because its weight on $E_{a}^{+}$will be random even in the case of an unconditionally riskless asset. Figure 8 shows the USFs with and without a safe asset.

Let us turn to the potential duality between the URF and the USF. As we have just seen, the latter is given by a single element, $q_{a}^{*}$, which is fully traded, with a return $S_{a}^{*}$ (defined in (44)) that clearly belongs to the URF. But $q_{a}^{*}$ and $S_{a}^{*}$ are only conditionally proportional, which means that even in the case of an unconditionally riskless asset we cannot establish a relationship between the slope of the URF and the slope of the ray that goes from the origin to $q_{a}^{*}$ in $[E(m), \sqrt{\operatorname{Var}(m)}]$ space. More formally, we will generally have that

$$
S R_{U}=\frac{\left|E\left(S_{a}^{*}\right)-S_{0}\right|}{\operatorname{Var}^{1 / 2}\left(S_{a}^{*}\right)} \neq \frac{\operatorname{Var}^{1 / 2}\left(q_{a}^{*}\right)}{E\left(q_{a}^{*}\right)}
$$

where the middle expression can be better described as a pseudo-Sharpe ratio, ${ }^{19}$ since it is based on the unconditional moments of $S_{a}^{*}$ in excess of the "safe" extended return $S_{0}$. Therefore, one must be careful in extending to unconditional frontiers of actively managed portfolios the geometrical relationship obtained by Hansen and Jagannathan (1991) in terms of pseudo-Sharpe ratios of passive portfolios. In particular, such a relationship does not hold between the elements of the URF and the USF, which simply reflects the fact that these two frontiers are not dual, as indicated by Proposition 2 .

\subsection{Extended Frontiers}

The elements of the ERF solve the same problem as in (19), except that $p$ is allowed to belong to the enlarged conditional span $Q_{a}$. In addition, they will lie along two straight lines in $[\sqrt{\operatorname{Var}(p)}, E(p)]$ space that cross on the vertical axis at a point with mean $S_{0}$ regardless

\footnotetext{
${ }^{19} S R_{U}$ was already defined by Jagannathan (1996). Nevertheless, his result relating the pseudo-Sharpe ratio of the URF with the Sharpe-ratio of the CRF

$$
\frac{1}{1+S R_{U}^{2}}=E\left[\frac{1}{1+S R_{1}^{2}}\right]
$$

does not necessarily hold unless the safe asset is unconditionally riskless, in which case $S R_{U}$ will be a proper Sharpe ratio.
} 
of whether the riskless asset is unconditionally safe or not. In either case, the ERF will not generally share any point with $p_{E}(\bar{\nu})$ in $(20)$. Figure 8 illustrates the ERFs with and without a safe asset.

As expected, the single element of the USF $q_{a}^{*}$ defined in (43) has a dual element on the $\mathrm{ERF}$, which is given by

$$
S_{e}^{*}=\frac{1}{E\left(q_{a}^{* 2}\right)} q_{a}^{*}
$$

In addition, there is a clear connection between slopes of the return and SDF frontiers because both elements are unconditionally proportional. Specifically,

$$
S R_{E}=\frac{\left|E\left(S_{e}^{*}\right)-S_{0}\right|}{\operatorname{Var}^{1 / 2}\left(S_{e}^{*}\right)}=\frac{\operatorname{Var}^{1 / 2}\left(q_{a}^{*}\right)}{E\left(q_{a}^{*}\right)},
$$

which means that the constant pseudo-Sharpe ratio $^{20}$ of the elements of the ERF will be equal to the slope of a ray from the origin to the single element of the USF. This geometry is analogous to the one illustrated in Figure 7.

Let us turn to the ESF. To do so, we must first define the subspace of constant-cost portfolios $Q_{e} \supset P_{e}$, and obtain the extended representing portfolios $q_{e}^{+}$and $q_{e}^{*}$ in that subspace. Then, we simply need to translate (28) to this context by defining for instance

$$
q_{e}^{*}=\frac{1}{E\left(S_{a}^{* 2}\right)} S_{a}^{*}
$$

A key novel feature of this extended set-up is that while the residual in the conditional projection of $x_{0}$ onto $Q_{a}$ is 0 , the residual from the unconditional projection of $x_{0}$ onto $Q_{e}$ $\left(=1-q_{e}^{+}\right)$is not necessarily 0 because the safe asset will have a random cost unless it is unconditionally riskless.

The elements of the ESF solve the same problem as in (25), but with the additional "pricing" constraint

$$
E\left(m x_{0} \mid G_{1}\right)=h_{1} c_{01}
$$

Nevertheless, this pricing constraint is not generally enough to pin down a particular $\bar{c}$, and hence the ESF will contain infinite points. However, when there is an unconditionally riskless asset, extended SDFs must price a unit payoff correctly on average, in which case the ESF will be given by the single point $q_{e}^{*}$. This point will be such that $E\left(q_{e}^{*}\right)=c_{0}$ and $\operatorname{Var}\left(q_{e}^{*}\right) \leq \operatorname{Var}\left(q_{a}^{*}\right)$. In either case, the ESF with and without a safe asset will not generally share any points, as illustrated in Figure 8.

\footnotetext{
${ }^{20}$ Note that $S R_{E}$ will not a proper Sharpe ratio even if $R_{0}=S_{0}$ because $S_{e}^{*}$ is not a proper return.
} 
As for the duality between the ESF and the URF, it is easy to see that the ESF will always be fully traded, and moreover, that its return will be $S_{a}^{*}$, which is defined in (44). Hence, if we think in terms of returns the ESF effectively consists of the single element $S_{a}^{*}$, which also belongs to the URF. However, transforming an element of the ESF into a return will require a conditional scaling unless we specifically focus on $q_{e}^{*}$. Not surprisingly,

$$
S R_{U}=\frac{\left|E\left(S_{a}^{*}\right)-S_{0}\right|}{\operatorname{Var}^{1 / 2}\left(S_{a}^{*}\right)}=\frac{\operatorname{Var}^{1 / 2}\left(q_{e}^{*}\right)}{E\left(q_{e}^{*}\right)},
$$

which means that the pseudo-Sharpe ratio of $S_{a}^{*}$ is equal to the slope of a ray from the origin to $q_{e}^{*}$ in $[E(m), \sqrt{\operatorname{Var}(m)}]$ space, so that the geometry is analogous to the one in Figure 7.

If there is an unconditionally riskless asset, then $S R_{U} \leq S R_{E}$, which means that a bound on the volatility of SDFs obtained from $S R_{U}$ might be too low, and a pseudo-Sharpe ratio obtained from $q_{a}^{*}$ might be too high. As a result, the intertemporal marginal rate of substitution in consumption of a specific CCAPM may look volatile enough from the perspective of $S R_{U}$ even though it would be insufficiently volatile to match $q_{a}^{*}$ (cf. Cochrane (2001, sect 21.1).

\subsection{Passive Frontiers}

The unconditional span of $\mathbf{y}$, i.e. $Q=\langle\mathbf{y}\rangle$, defines an alternative payoff space $Q_{a} \supseteq Q \supset P$, which is the relevant one for passive strategies. In this context, we can define the passive mean representing portfolio $q^{+}$as the unconditional projection of $x_{0}$ onto $Q$, and the associated cost representing portfolio $q^{*}$ as the unconditional projection of any passive SDF onto $Q$ (trivially $m_{P}\left(c_{0}\right)$ from $\left.(36)\right)$, so that

$$
q^{+}=1, \quad q^{*}=p^{*}+\left[\frac{c_{0}-\mathrm{A}}{1-\mathrm{B}}\right] E^{+}
$$

Once again, the residual in the unconditional projection of $x_{0}$ onto $Q$ will be 0 .

The PRF solves a problem analogous to the one in (31), with the only difference that $p \in Q$. Not surprisingly, its elements will lie along two straight lines in $[\sqrt{\operatorname{Var}(p)}, E(p)]$ space that cross on the vertical axis at a point whose mean is $S_{0}$ defined in (42), regardless of whether the riskless asset is unconditionally safe or not. In either case, the PRF will always share a point with the $p_{P}(\bar{\nu})^{\prime} s$ in $(32)$.

On the other hand, the elements of the PSF will be given by the solution to a problem analogous to (35), with the only change that the pricing constraints become $E(m \mathbf{y})=\mathbf{d}$. But as expected, the point $\bar{c}=c_{0}$ is the only choice compatible with the pricing constraints, which 
implies that the PSF is a singleton. In this sense, Hansen and Jagannathan (1991) show that this single element will be $q^{*}$, which is equal to $m_{P}\left(c_{0}\right)$, where $m_{P}(\bar{c})$ was defined in (36). Importantly, $q^{*}$ has exactly the same mean as the single element of the USF although it will lie below it in $[E(m), \sqrt{\operatorname{Var}(m)}]$ space.

Finally, Hansen and Jagannathan (1991) also show that the point on the PRF given by

$$
S^{*}=\frac{1}{E\left(q^{* 2}\right)} q^{*}
$$

has a pseudo-Sharpe ratio that equals the slope of a ray from the origin to $q^{*}$ in $[E(m), \sqrt{\operatorname{Var}(m)}]$ space, so that

$$
S R_{P}=\frac{\left|E\left(S^{*}\right)-S_{0}\right|}{\operatorname{Var}^{1 / 2}\left(S^{*}\right)}=\frac{\operatorname{Var}^{1 / 2}\left(q^{*}\right)}{E\left(q^{*}\right)} .
$$

Figure 9 shows the passive, unconditional and extended frontiers for portfolios and SDF's for the case of an unconditionally safe asset and $\mathbf{x}$ given by a return vector. Obviously, we can also add managed portfolios to this set-up. For instance, Ferson and Siegel (2006) use constant cost managed portfolios in the computation of $S R_{P}$.
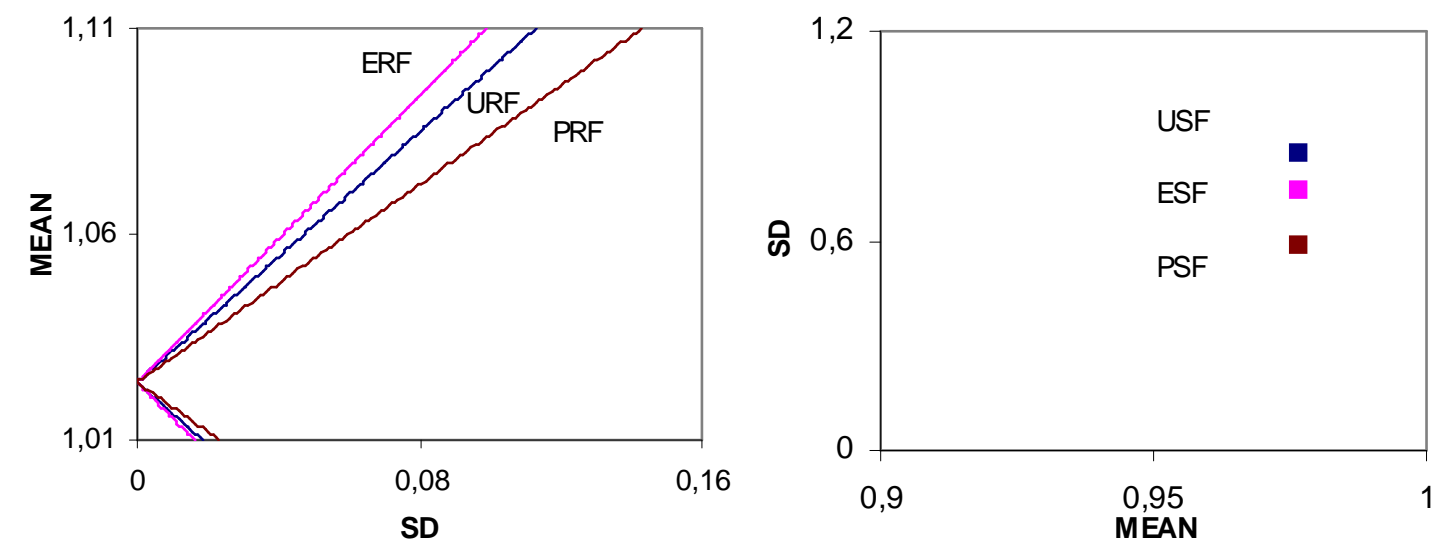

Figure 9: Passive frontiers with an unconditionally riskless asset, jointly with unconditional and extended frontiers. Portfolio frontiers on the left and SDF frontiers on the right.

\section{Other Special Cases}

As we mentioned before, there are two other special cases in which mean-variance frontiers adopt a particularly simple form. One such case arises when all expected payoffs are conditionally proportional to their prices, with a common scalar factor of proportionality. The other one occurs when all the primitive assets are arbitrage portfolios. 


\subsection{Prices Proportional to Expected Payoffs}

Although this situation is typically associated with the equilibrium of an economy with a risk-neutral agent, it also arises when $N=1$, an example that was used by Ferson and Siegel (2003) and Bekaert and Liu (2004) to differentiate their papers. ${ }^{21}$ Given that in either case $p_{a}^{+}=k_{1} p_{a}^{*}$, with $k_{1} \in I_{1}$, the geometry of the return and SDF frontiers turns out to be the mirror image of the safe asset case if we interchange the shapes of the return and SDF frontiers. In particular, while the main implication of the existence of a safe asset was that $1-q_{a}^{+}=0$, with the additional feature that $1-q_{e}^{+}=0$ if the safe asset asset was unconditionally riskless, the main implication now is that $A_{a}^{+}$defined in (9) will be 0 , with the additional feature that $A_{e}^{+}$defined in (22) will also be 0 if expected payoffs are unconditionally proportional to their prices, i.e. if $k_{1}=k \in \mathbb{R}$.

In this context, the $\mathrm{CRF} p_{C}\left(\bar{v}_{\mathrm{I}}\right)$ will be given by the single element $R_{a}^{*}$, which was defined in (8). On the other hand, the risky part of the CSF $m_{C}\left(\bar{c}_{1}\right)$ can be obtained by conditionally scaling $R_{a}^{*}$. As a result, for each signal value the CSF will be represented by two straight lines in $\left[E\left(m \mid G_{1}\right), \sqrt{\operatorname{Var}\left(m \mid G_{1}\right)}\right]$ space that touch at the horizontal axis when $\bar{c}_{\mathbf{1}}=k_{\mathbf{1}}^{-1}$. The duality between the straight lines that characterise $m_{C}\left(\bar{c}_{1}\right)$ and the point $p_{C}\left(\bar{v}_{\mathbf{1}}\right)$ relies on the fact that the return corresponding to the traded part of any $m_{C}\left(\bar{c}_{1}\right)$ is always $R_{a}^{*}$.

A similar type of duality applies for the pairs URF/ESF and ERF/USF. Specifically, the $\mathrm{URF} p_{U}(\bar{v})$ will be given by the same single point $R_{a}^{*}$ for the reasons explained when we discussed the CRF in the presence of a riskless asset. The ESF $m_{E}(\bar{c})$ is now given by two straight lines in $[E(m), \sqrt{\operatorname{Var}(m)}]$ space that touch the horizontal axis at $\bar{c}=E^{-1}\left(k_{1}\right)$ because the scaling of $R_{a}^{*}$ is nonrandom.

In contrast, the USF $m_{U}(\bar{c})$ and the $\operatorname{ERF} p_{E}(\bar{v})$ do not show any relevant changes with respect to the general case. However, if $k_{1}=k \in R$, then the USF will be given by two straight lines in $[E(m), \sqrt{\operatorname{Var}(m)}]$ space that touch the horizontal axis at $\bar{c}=k^{-1}$, and the ERF will be the single point $R_{e}^{*}$ defined in (21) with $E\left(R_{e}^{*}\right)=E\left(R_{a}^{*}\right)=k$.

The situation is slightly different when we consider passive frontiers. While in the safe asset case $1-q^{+}=0$ irrespective of whether or not the safe asset is unconditionally riskless, here passive frontiers do not show any relevant changes unless $k_{1}=k \in \mathbb{R}$ or $N=1$, in which two

\footnotetext{
${ }^{21}$ Intuitively, the approach used by Ferson and Siegel (2003) to obtain SDF bounds cannot exploit the existence of conditioning information when $N=1$ because the elements of the URF (14) are constrained to have constant (unit) cost. See equation (24).
} 
cases $A^{+}$(defined in (34)) is equal to 0 . Therefore, it is only in those circumstances that we will have the mirror image situation to the safe asset case, in that the PRF $p_{P}(\bar{v})$ will collapse to the point $R^{*}$, while the PSF $m_{P}(\bar{c})$ will given by two straight lines in $[E(m), \sqrt{\operatorname{Var}(m)}]$ space.

\subsection{Arbitrage Portfolios}

Let us finally study the situation in which all primitive assets are arbitrage portfolios, so that $\mathbf{c}_{1}=\mathbf{0}$. This case is quite common in empirical work, as asset payoffs are routinely transformed into excess returns in the presence of a (conditionally) riskless asset. From the point of view of mean-variance frontiers, the main implication of dealing with arbitrage portfolios is that the active and passive cost representing portfolios defined in (4) and (30), respectively, are both zero. Therefore, there is one-fund spanning in every frontier and consequently, all of them can be represented by straight lines that start from the origin in the appropriate mean-standard deviation space.

More specifically, since the cost of any portfolio of $\mathbf{x}$ is 0 in this case, the portfolio frontiers problems can be defined as before (see problems (6), (14),(19), and (31)) after dropping the cost constraints. That is, each problem consists now in minimising the second moment of portfolios given a constraint on their first moment. As a result, the URF and ERF coincide in this context since their only difference was the cost constraint. The CRF is constructed by a conditional scaling of $p_{a}^{+}$, the URF by an unconditional scaling of $p_{a}^{+}$, and the PRF by an unconditional scaling of $p^{+}$.

Interestingly, if the $N$ arbitrage portfolios under analysis correspond to the excess returns of $N$ risky assets over an unconditionally riskless asset, the slope of the URF/ERF discussed in the previous paragraph will coincide with the slope of the URF discussed at the beginning of section 7.2 , which combines the original $N$ risky returns and the unconditionally safe asset. Therefore, the maximum unconditional Sharpe ratios attainable in both situations will also be the same, and will exceed the unconditional Sharpe ratios in Bekaert and Liu (2003) and Abhyankar, Basu and Stremme (2007) mentioned in the same section.

On the other hand, the pricing constraints of the SDF frontiers (see problems (10), (16),(25), and (35)) imply that any valid SDF must be orthogonal to $\mathbf{x}$. Moreover, since $P_{a}=P_{e}$ in this context, the USF and ESF will also coincide. The CSF is constructed by a conditional scaling of $E_{a}^{+}$, the USF by an unconditional scaling of $E_{a}^{+}$, and the PSF by an unconditional scaling of $E^{+}$, where $E_{a}^{+}$and $E^{+}$were defined in (12) and (37), respectively. Obviously, we can also 
add managed portfolios to this set-up. For instance, Bekaert and Hodrick (1992) use managed portfolios of zero cost in the computation of the PSF's slope.

\section{Conclusion}

We use representing portfolios to analyse four mean-variance frontiers for SDF-like objects, which differ in the type of portfolios that they price. One frontier relies on conditional moments, while the other three, which are the usual focus of empirical work, rely on unconditional moments. Specifically, we consider:

1) The USF introduced by Gallant, Hansen, and Tauchen (1990), which computes the highest lower bound on the variance of SDFs, i.e. those univariate random variables that correctly price any portfolio with weights that may depend on conditioning information. This frontier coincides with the one discussed by Bekaert and Liu (2004).

2) The ESF, which provides the highest lower bound on the variance of those univariate random variables that price on average any portfolio with weights that may depend on conditioning information but whose cost is constant. These are not necessarily valid SDFs because they may not price correctly portfolios whose cost is a function of the conditioning information. This frontier coincides with the one advocated by Ferson and Siegel (2003).

3) The PSF introduced by Hansen and Jagannathan (1991), which computes the highest lower bound on the variance of those univariate random variables that price on average any portfolio with constant weights. Again, these are not necessarily valid SDFs either, as they may not price correctly portfolios with time-varying weights.

Given these precise characterisations, it is not surprising that the USF will always be above both the ESF and PSF in the usual mean-standard deviation space. In contrast, depending on whether the payoffs under consideration have constant or random cost, the PSF may be above or below the ESF, either over its entire domain, or over some part.

In this context, we explicitly characterise the random variables for which the appropriate dual portfolio objects are themselves frontiers, as opposed to mere volatility bounds. Using the same order as before, these dual objects are a frontier for conditional moments, as well as:

1) The ERF, which for each level of expected return computes the highest lower bound on the variance of any portfolio with weights that may depend on conditioning information whose price is one on average. Thus, the USF delivers interesting constraints on asset pricing models, 
but its dual (ERF) does not deliver interesting risk-return trade offs.

2) The URF introduced by Hansen and Richard (1987), which for each level of expected return computes the highest lower bound on the variance of any portfolio with weights that may depend on conditioning information but whose price is always one. Thus, the URF delivers interesting risk-return trade offs, but its dual (ESF) does not deliver equally interesting constraints on asset pricing models.

3) The PRF mentioned by Hansen and Jagannathan (1991), which for each level of expected return computes the highest lower bound on the variance of any portfolio with constant weights whose price is one on average. The PRF and its dual (PSF) are easy to implement but in general they are not the relevant objects to study regarding either risk-return trade-offs or constraints on asset pricing models.

Given these precise characterisations, it is not surprising that the ERF will always be to the left of both the URF and PRF in the usual mean-standard deviation space. In contrast, depending on whether the payoffs under consideration have constant or random cost, the PRF may be above or below the URF, either over its entire domain, or over some part.

Most empirical work on unconditional mean-variance frontiers relies on passive strategies of managed portfolios as a way of approximating the complexity of active strategies without running the risk of misspecifying the conditional distribution of asset returns. In this context, we show that if we used all the relevant (unrestricted) managed portfolios, then we would generate the USF. In the more plausible situation in which a researcher only uses some of them, she will generate a frontier between the PSF based on returns alone and the USF, which might still improve upon the ESF. But if she focuses on managed portfolios of constant cost, then the PSF converges to the ESF instead of the USF as the number of managed portfolios increases.

Then, our duality results imply that the use of all the relevant (unrestricted) managed portfolios would deliver the ERF, not the URF. In the more plausible situation in which a researcher only uses some of them, she will generate a frontier between the PRF based on returns alone and the ERF. But if she focuses on managed portfolios of constant cost, then the PRF converges to the URF instead of the ERF as the number of managed portfolios increases.

Therefore, an important empirical implication of our analysis is that a researcher who is interested in asset pricing questions should use unrestricted managed portfolios to approximate the unconditional SDF frontier (USF). In contrast, a researcher interested in portfolio choice 
questions should use managed portfolios of constant cost to approximate the unconditional return frontier (URF). By construction, though, passive frontiers of managed portfolios cannot approximate the conditional frontiers CRF and CSF.

Finally, we also study some special cases, the most important being the presence of an asset which is either conditionally or unconditionally riskless. In that case, we show that the geometric interpretation of duality in terms Sharpe ratios in Hansen and Jagannathan (1991) applies to their specific set-up of unconditional moments and passive strategies, and that we must again be careful in extending that result to other frameworks. For instance, the intertemporal marginal rate of substitution in consumption of a specific CCAPM may look volatile enough from the perspective of the Sharpe ratio on the URF even though it would be insufficiently volatile to match the USF.

We also show that there are some other results that are sometimes taken for granted, but which may also fail. For instance, a tangency portfolio does not exist on the URF and ERF irrespective of whether the safe asset is conditionally or unconditionally riskless. As a result, the Sharpe ratios that Bekaert and Liu (2003) and Abhyankar, Basu and Stremme (2007) consider must be interpreted with some care, as they relate to passive strategies that combine an unconditionally riskless asset (traded or fictitious) with a portfolio on the URF of risky assets alone.

The results in this paper are also useful to develop testing procedures related to those meanvariance frontiers that utilise conditioning information, as illustrated by Peñaranda and Sentana (2006) in the context of mean-variance spanning tests. 


\section{References}

Abhyankar, A., D. Basu, and A. Stremme (2007): Portfolio Efficiency and Discount Factor Bounds with Conditioning Information: An Empirical Study, Journal of Banking 6 Finance 31, 419-437.

Bansal, R., M. Dahlquist, and C.R. Harvey (2004): Dynamic Strategies and Portfolio Choice, NBER WP 10820.

Bekaert, G., and R.J. Hodrick (1992): Characterizing Predictable Components in Excess Returns on Equity and Foreign Exchange Markets, Journal of Finance 47, 467-509.

Bekaert, G.,and J. Liu (2004): Conditioning Information and Variance Bounds on Pricing Kernels, Review of Financial Studies 17, 339-378.

Bekaert, G., and M.S. Urias (1996): Diversification, Integration and Emerging Market Closed-End Funds, Journal of Finance 51, 835-869.

Brandt, M.W., and P. Santa-Clara (2006): Dynamic Portfolio Selection by Augmenting the Asset Space, Journal of Finance 61, 2187-2217.

Chamberlain, G. and M. Rothschild (1983): Arbitrage, Factor Structure, and Mean-Variance Analysis on Large Asset Markets, Econometrica 51, 1281-1304.

Cochrane, J.H. (2001): Asset Pricing, Princeton University Press.

De Roon, F.A., and T.E. Nijman (2001): Testing for Mean-Variance Spanning: A Survey, Journal of Empirical Finance 8, 111-155.

De Santis, G. (1995): Volatility Bounds for Stochastic Discount Factors: Tests and Implications from International Financial Markets, mimeo, University of Southern California.

Ferson, W.E., and A.F. Siegel (2001): The Efficient Use of Conditioning Information in Portfolios, Journal of Finance 56, 967-982.

Ferson, W.E., and A.F. Siegel (2003): Stochastic Discount Factor Bounds with Conditioning Information, Review of Financial Studies 16, 567-595.

Ferson, W.E., and A.F. Siegel (2006): Testing Portfolio Efficiency with Conditioning Information, NBER Working Paper No. 12098.

Gallant, A.R., L.P. Hansen and G. Tauchen (1990): Using Conditional Moments of Asset Payoffs to Infer the Volatility of Intertemporal Marginal Rates of Substitution, Journal of Econometrics 45, 141-179. 
Hansen, L.P., and R. Jagannathan (1991): Implications of Security Market Data for Models of Dynamic Economies, Journal of Political Economy 99, 225-262.

Hansen, L.P., and S.F. Richard (1987): The Role of Conditioning Information in Deducing Testable Restrictions Implied by Dynamic Asset Pricing Models, Econometrica 55, 587-613.

Jagannathan, R. (1996): Relation between the Slopes of the Conditional and Unconditional Mean-Standard Deviation Frontiers of Asset Returns, in S. Saito, K. Sawaki, and K. Kubota (eds.) Modern Portfolio Theory and its Applications, Center for Academic Societies, Osaka.

Markowitz, H. (1952): Portfolio Selection, Journal of Finance 7, 77-99.

Peñaranda, F., and E. Sentana (2006): Spanning Tests in Return and Stochastic Discount Factor Mean-Variance Frontiers: A Unifying Approach, mimeo.

Sentana, E. (2005): Least Squares Predictions and Mean-Variance Analysis, Journal of Financial Econometrics 3, 56-78. 


\section{Appendix}

\section{Proofs}

\section{Lemma 1:}

If we use equation (24) to represent $P_{e}$ then the definition of an extended SDF $m$ is equivalent to

$$
E\left[m\left(R_{1} w+\left(\mathbf{x}_{-1}-R_{1} \mathbf{c}_{1,-1}\right)^{\prime} \mathbf{w}_{\mathbf{1},-1}\right)\right]=w, \quad \forall w \in \mathbb{R}, \quad \forall \mathbf{w}_{1,-1} \in I_{1} .
$$

This is true if and only if

$$
\begin{gathered}
E\left(m R_{1}\right)=1, \\
E\left(m\left(\mathbf{x}_{-1}-R_{1} \mathbf{c}_{\mathbf{1},-1}\right) \mid G_{1}\right)=\mathbf{0} .
\end{gathered}
$$

The former condition can be re-written as

$$
E\left(m R_{1} \mid G_{1}\right)=h_{1} \in I_{1}, \quad E\left(h_{1}\right)=1
$$

and the last condition as

$$
E\left(m \mathbf{x}_{-1} \mid G_{1}\right)=E\left(m R_{1} \mid G_{1}\right) \mathbf{c}_{1,-1}=h_{1} \mathbf{c}_{1,-1}
$$

Therefore, $m$ is an extended SDF if and only if

$$
\begin{aligned}
E\left(m \mathbf{x} \mid G_{1}\right) & =h_{1} \mathbf{c}_{1}, \quad h_{1} \in I_{1} \\
E\left(h_{1}\right) & =1,
\end{aligned}
$$

which completes the proof.

\section{Proposition 1:}

a) We can express the CSF (11) as

$$
m_{C}\left(\bar{c}_{1}\right)=\left[\mathrm{C}_{1}-\varpi_{1}\left(\bar{c}_{1}\right) \mathrm{A}_{1}\right] R_{a}^{*}-\varpi_{1}\left(\bar{c}_{1}\right) A_{a}^{+}+\varpi_{1}\left(\bar{c}_{1}\right) .
$$

Then we only have to rescale its risky part by its conditional cost $C_{1}-\varpi_{1}\left(\bar{c}_{1}\right) A_{1}$ when it is not 0 to get a return on the CRF (7). Specifically,

$$
R_{a}^{*}-\frac{\varpi_{1}\left(\bar{c}_{1}\right)}{\mathrm{C}_{1}-\varpi_{1}\left(\bar{c}_{1}\right) \mathrm{A}_{1}} A_{a}^{+}
$$

is the element on the CRF such that

$$
\omega_{1}\left(\bar{\nu}_{1}\right)=-\frac{\varpi_{1}\left(\bar{c}_{1}\right)}{\mathrm{C}_{1}-\varpi_{1}\left(\bar{c}_{1}\right) \mathrm{A}_{1}} .
$$


b) We can express the CRF (7) as

$$
p_{C}\left(\bar{\nu}_{1}\right)=\left(\frac{1-\omega_{1}\left(\bar{\nu}_{1}\right) \mathrm{A}_{1}}{\mathrm{C}_{1}}\right) p_{a}^{*}+\omega_{1}\left(\bar{\nu}_{1}\right) p_{a}^{+} .
$$

Hence, when $1-\omega_{1}\left(\bar{\nu}_{1}\right) \mathrm{A}_{1} \neq 0$, we can rescale $p_{C}\left(\bar{\nu}_{1}\right)$ to get the traded part of a SDF on the CSF (11). In particular,

$$
p_{a}^{*}+\frac{\omega_{1}\left(\bar{\nu}_{1}\right) \mathrm{C}_{1}}{1-\omega_{1}\left(\bar{\nu}_{1}\right) \mathrm{A}_{1}} p_{a}^{+}
$$

is the traded part of an element of the CSF such that

$$
\varpi_{1}\left(\bar{c}_{1}\right)=-\frac{\omega_{1}\left(\bar{\nu}_{1}\right) \mathrm{C}_{1}}{1-\omega_{1}\left(\bar{\nu}_{1}\right) \mathrm{A}_{1}}
$$

which completes the proof.

\section{Proposition 2:}

a) We can express the USF (17) as

$$
m_{U}(\bar{c})=\left[\mathrm{C}_{\mathbf{1}}-\varpi_{U}(\bar{c}) \mathrm{A}_{\mathbf{1}}\right] R_{a}^{*}-\varpi_{U}(\bar{c}) A_{a}^{+}+\varpi_{U}(\bar{c}) .
$$

We could rescale the risky part by its conditional cost $\mathrm{C}_{1}-\varpi_{U}(\bar{c}) \mathrm{A}_{1}$, when it is not 0 , to look for a dual point on the URF (15). Specifically,

$$
R_{a}^{*}-\frac{\varpi_{U}(\bar{c})}{\mathrm{C}_{1}-\varpi_{U}(\bar{c}) \mathrm{A}_{1}} A_{a}^{+}
$$

should be linked to $R_{a}^{*}+\omega_{U}(\bar{\nu}) A_{a}^{+}$.

b) We can express the URF (15) as

$$
p_{U}(\bar{\nu})=\left(\frac{1-\omega_{U}(\bar{\nu}) \mathrm{A}_{\mathbf{1}}}{\mathrm{C}_{\mathbf{1}}}\right) p_{a}^{*}+\omega_{U}(\bar{\nu}) p_{a}^{+}
$$

Hence, when $1-\omega_{U}(\bar{\nu}) \mathrm{A}_{\perp} \neq 0$, we could look for a dual point on the the USF (17). In particular,

$$
p_{a}^{*}+\frac{\omega_{U}(\bar{\nu}) \mathrm{C}_{1}}{1-\omega_{U}(\bar{\nu}) \mathrm{A}_{1}} p_{a}^{+}
$$

should be linked to $p_{a}^{*}-\varpi_{U}(\bar{c}) p_{a}^{+}$. 


\section{Proposition 3:}

We can decompose any portfolio $p$ satisfying the constraints in (19) as its unconditional projection onto $\left\langle R_{e}^{*}, A_{e}^{+}\right\rangle=\left\langle p_{a}^{+}, p_{a}^{*}\right\rangle$ plus some unconditionally orthogonal residual $u$. Specifically,

$$
\begin{gathered}
p=\tilde{p}+u \\
\tilde{p}=\left(\begin{array}{c}
R_{e}^{*} \\
A_{e}^{+}
\end{array}\right)^{\prime} E^{-1}\left[\begin{array}{cc}
R_{e}^{* 2} & R_{e}^{*} A_{e}^{+} \\
R_{e}^{*} A_{e}^{+} & A_{e}^{+2}
\end{array}\right] E\left[\begin{array}{c}
p R_{e}^{*} \\
p A_{e}^{+}
\end{array}\right] \\
=\left(\begin{array}{c}
R_{e}^{*} \\
A_{e}^{+}
\end{array}\right)^{\prime}\left[\begin{array}{cc}
E\left(R_{e}^{* 2}\right) & 0 \\
0 & E\left(A_{e}^{+}\right)
\end{array}\right]^{-1}\left[\begin{array}{c}
1 / E\left(\mathrm{C}_{\mathbf{1}}\right) \\
\bar{\nu}-E\left(\mathrm{~A}_{\mathbf{1}}\right) / E\left(\mathrm{C}_{\mathbf{1}}\right)
\end{array}\right] .
\end{gathered}
$$

Hence

$$
\tilde{p}=\frac{1 / E\left(\mathrm{C}_{1}\right)}{E\left(R_{e}^{* 2}\right)} R_{e}^{*}+\frac{\bar{\nu}-E\left(\mathrm{~A}_{1}\right) / E\left(\mathrm{C}_{1}\right)}{E\left(A_{e}^{+}\right)} A_{e}^{+}=R_{e}^{*}+\omega_{E}(\bar{\nu}) A_{e}^{+},
$$

where $\omega_{E}(\bar{\nu})$ is defined as in $(20)$.

It is easy to see that $\tilde{p}$ satisfies the constraints in (19): First,

$$
E(\tilde{p})=E\left(R_{e}^{*}\right)+\omega_{E}(\bar{\nu}) E\left(A_{e}^{+}\right)=\frac{E\left(\mathrm{~A}_{1}\right)}{E\left(\mathrm{C}_{1}\right)}+\left[\frac{\bar{\nu}-E\left(\mathrm{~A}_{1}\right) / E\left(\mathrm{C}_{1}\right)}{E\left(A_{e}^{+}\right)}\right] E\left(A_{e}^{+}\right)=\bar{\nu},
$$

and also

$$
C(\tilde{p})=C\left(R_{e}^{*}\right)+\omega_{E}(\bar{\nu}) C\left(A_{e}^{+}\right)=1+\omega_{E}(\bar{\nu}) 0=1,
$$

Finally, by construction,

$$
E\left(p^{2}\right)=E\left(\tilde{p}^{2}\right)+E\left(u^{2}\right)
$$

and hence the solution to (19) is $\tilde{p}$, which is exactly $p_{E}(\bar{\nu})$ in $(20)$.

\section{Proposition 4:}

a) We can express the USF (17) as

$$
m_{U}(\bar{c})=\left(E\left(\mathrm{C}_{\mathbf{1}}\right)-\varpi_{U}(\bar{c}) E\left(\mathrm{~A}_{\mathbf{1}}\right)\right) R_{e}^{*}-\varpi_{U}(\bar{c}) A_{e}^{+}+\varpi_{U}(\bar{c}) .
$$

Then we only have to rescale the risky part by its average cost $E\left(\mathrm{C}_{1}\right)-\varpi_{U}(\bar{c}) E\left(\mathrm{~A}_{1}\right)$ when it is not 0 to get an extended return on the ERF (20). Specifically,

$$
R_{e}^{*}-\frac{\varpi_{U}(\bar{c})}{E\left(\mathrm{C}_{\mathbf{1}}\right)-\varpi_{U}(\bar{c}) E\left(\mathrm{~A}_{\mathbf{1}}\right)} A_{e}^{+}
$$

is equal to an element on the ERF for the corresponding $\omega_{E}(\bar{\nu})$. 
b) We can represent the ERF (20) as

$$
p_{E}(\bar{\nu})=\left[\frac{1-\omega_{E}(\bar{\nu}) E\left(\mathrm{~A}_{1}\right)}{E\left(\mathrm{C}_{1}\right)}\right] p_{a}^{*}+\omega_{E}(\bar{\nu}) p_{a}^{+} .
$$

Hence, for each $\omega_{E}(\bar{\nu})$ such that $1-\omega_{E}(\bar{\nu}) E\left(\mathrm{~A}_{1}\right) \neq 0$, this can be re-written as the traded the traded part of a SDF on the USF (17). In particular,

$$
p_{a}^{*}+\frac{\omega_{E}(\bar{\nu}) E\left(\mathrm{C}_{1}\right)}{1-\omega_{E}(\bar{\nu}) E\left(\mathrm{~A}_{1}\right)} p_{a}^{+}
$$

is equal to an element on the USF for the corresponding $\varpi_{U}(\bar{c})$.

\section{Proposition 5:}

We can decompose any extended SDF $m$ satisfying the constraints in (25) as its unconditional projection onto $\left\langle p_{e}^{*}, E_{e}^{+}\right\rangle$plus some unconditionally orthogonal residual $u$. In particular,

$$
\begin{gathered}
m=\tilde{m}+u \\
\tilde{m}=\left(\begin{array}{c}
p_{e}^{*} \\
E_{e}^{+}
\end{array}\right)^{\prime} E^{-1}\left[\begin{array}{cc}
p_{e}^{* 2} & p_{e}^{*} E_{e}^{+} \\
p_{e}^{*} E_{e}^{+} & E_{e}^{+2}
\end{array}\right] E\left[\begin{array}{c}
m p_{e}^{*} \\
m E_{e}^{+}
\end{array}\right] \\
=\left(\begin{array}{c}
p_{e}^{*} \\
E_{e}^{+}
\end{array}\right)^{\prime}\left[\begin{array}{cc}
E\left(p_{e}^{* 2}\right) & 0 \\
0 & E\left(E_{e}^{+}\right)
\end{array}\right]^{-1}\left[\begin{array}{c}
1 / E\left(\mathrm{~F}_{\mathbf{1}}\right) \\
\bar{c}-E\left(\mathrm{D}_{\mathbf{1}}\right) / E\left(\mathrm{~F}_{\mathbf{1}}\right)
\end{array}\right] .
\end{gathered}
$$

If we define $\varpi_{E}(\bar{c})$ as in (26) then we can write

$$
\tilde{m}=\frac{1 / E\left(\mathrm{~F}_{1}\right)}{E\left(p_{e}^{* 2}\right)} p_{e}^{*}+\frac{\bar{c}-E\left(\mathrm{D}_{1}\right) / E\left(\mathrm{~F}_{1}\right)}{E\left(E_{e}^{+}\right)} E_{e}^{+}=p_{e}^{*}+\varpi_{E}(\bar{c}) E_{e}^{+} .
$$

It is easy to see that $\tilde{m}$ satisfies the constraints in (25): First,

$$
E(\tilde{m})=E\left(p_{e}^{*}\right)+\varpi_{E}(\bar{c}) E\left(E_{e}^{+}\right)=\frac{E\left(\mathrm{D}_{1}\right)}{E\left(\mathrm{~F}_{1}\right)}+\left[\frac{\bar{c}-E\left(\mathrm{D}_{1}\right) / E\left(\mathrm{~F}_{1}\right)}{E\left(E_{e}^{+}\right)}\right] E\left(E_{e}^{+}\right)=\bar{c},
$$

and also

$$
\begin{gathered}
E\left(\tilde{m} \mathbf{x} \mid G_{1}\right)=E\left(p_{e}^{*} \mathbf{x} \mid G_{1}\right)+\varpi_{E}(\bar{c}) E\left(E_{e}^{+} \mathbf{x} \mid G_{1}\right) \\
=\frac{1}{E\left(\mathrm{~F}_{1}\right)} E\left(R_{a}^{*} \mathbf{x} \mid G_{1}\right)+\varpi_{E}(\bar{c})\left[E\left(\left(1-A_{a}^{+}\right) \mathbf{x} \mid G_{1}\right)-\frac{E\left(\mathrm{D}_{1}\right)}{E\left(\mathrm{~F}_{1}\right)} E\left(R_{a}^{*} \mathbf{x} \mid G_{1}\right)\right] \\
=h_{1} \mathbf{c}_{1}, \quad h_{1}=\left(\frac{1-\varpi_{E}(\bar{c}) E\left(\mathrm{D}_{1}\right)}{E\left(\mathrm{~F}_{1}\right)}\right) \mathrm{F}_{1}+\varpi_{E}(\bar{c}) \mathrm{D}_{1},
\end{gathered}
$$

with

$$
E\left(h_{1}\right)=\left(\frac{1-\varpi_{E}(\bar{c}) E\left(\mathrm{D}_{1}\right)}{E\left(\mathrm{~F}_{1}\right)}\right) E\left(\mathrm{~F}_{1}\right)+\varpi_{E}(\bar{c}) E\left(\mathrm{D}_{1}\right)=1 .
$$

Finally, by construction,

$$
E\left(m^{2}\right)=E\left(\tilde{m}^{2}\right)+E\left(u^{2}\right)
$$

and hence the solution to (25) is $\tilde{m}$, which is exactly $m_{E}(\bar{c})$ in $(26)$. 


\section{Proposition 6:}

a) We can express the ESF (26) as

$$
m_{E}(\bar{c})=\left(\frac{1-\varpi_{E}(\bar{c}) E\left(\mathrm{D}_{\mathbf{1}}\right)}{E\left(\mathrm{~F}_{\mathbf{1}}\right)}\right) R_{a}^{*}-\varpi_{E}(\bar{c}) A_{a}^{+}+\varpi_{E}(\bar{c}) .
$$

Then we only have to rescale the risky part by its conditional cost $\left(1-\varpi_{E}(\bar{c}) E\left(\mathrm{D}_{1}\right)\right) / E\left(\mathrm{~F}_{1}\right)$ (which is constant) when it is different from 0 to get a return on the URF (15). Specifically,

$$
R_{a}^{*}-\frac{\varpi_{E}(\bar{c})}{\left(1-\varpi_{E}(\bar{c}) E\left(\mathrm{D}_{1}\right)\right) / E\left(\mathrm{~F}_{\mathbf{1}}\right)} A_{a}^{+}
$$

is equal to an element on the URF given by the corresponding $\omega_{U}(\bar{\nu})$.

b) We can express the URF (15) as

$$
p_{U}(\bar{\nu})=\left(\mathrm{F}_{1}-\omega_{U}(\bar{\nu}) \mathrm{D}_{1}\right) p_{a}^{*}+\omega_{U}(\bar{\nu}) p_{a}^{+} .
$$

Rescaling this expression by its average position on $p_{a}^{*}$ when $E\left(\mathrm{~F}_{1}\right)-\omega_{U}(\bar{\nu}) E\left(\mathrm{D}_{1}\right) \neq 0$, we construct the traded part of an extended SDF on the ESF (26). In particular,

$$
\left(E\left(\mathrm{~F}_{1}\right)-\omega_{U}(\bar{\nu}) E\left(\mathrm{D}_{1}\right)\right)^{-1}\left[\left(\mathrm{~F}_{1}-\omega_{U}(\bar{\nu}) \mathrm{D}_{1}\right) p_{a}^{*}+\omega_{U}(\bar{\nu}) p_{a}^{+}\right]
$$

is the traded part of an element on the ESF given by the corresponding $\varpi_{E}(\bar{c})$. 